\title{
Early Evolution of Stellar Groups and Clusters: Environmental Effects on Forming Planetary Systems
}

\author{
Fred C. Adams, ${ }^{1,2}$ Eva M. Proszkow, ${ }^{1}$ Marco Fatuzzo,${ }^{3}$ and Philip C. Myers ${ }^{4}$ \\ ${ }^{1}$ Michigan Center for Theoretical Physics, University of Michigan, Ann Arbor, MI 48109 \\ ${ }^{2}$ Astronomy Department, University of Michigan, Ann Arbor, MI 48109 \\ ${ }^{3}$ Physics Department, Xavier University, Cincinnati, OH 45207 \\ ${ }^{4}$ Harvard Smithsonian Center for Astrophysics, 60 Garden Street, Cambridge, MA 02138 \\ fca@umich.edu
}

\begin{abstract}
This paper studies the dynamical evolution of young groups/clusters, with $N=$ $100-1000$ members, from their embedded stage out to ages of $\sim 10$ Myr. We use $N$-body simulations to explore how their evolution depends on the system size $N$ and the initial conditions. Motivated by recent observations suggesting that stellar groups begin their evolution with subvirial speeds, this study compares subvirial starting states with virial starting states. Multiple realizations of equivalent cases (100 simulations per initial condition) are used to build up a robust statistical description of these systems, e.g., the probability distribution of closest approaches, the mass profiles, and the probability distribution for the radial location of cluster members. These results provide a framework from which to assess the effects of groups/clusters on the processes of star and planet formation, and to study cluster evolution. The distributions of radial positions are used in conjunction with the probability distributions of the expected FUV luminosities (calculated here as a function of cluster size $N$ ) to determine the radiation exposure of circumstellar disks. The distributions of closest approaches are used in conjunction with scattering cross sections (calculated here as a function of stellar mass using $\sim 10^{5}$ Monte Carlo scattering experiments) to determine the probability of disruption for newly formed solar systems. We use the nearby cluster NGC 1333 as a test case in this investigation. The main conclusion of this study is that clusters in this size range have only a modest effect on forming planetary systems. The interaction rates are low so that the typical solar system experiences a single encounter with closest approach distance $b \sim 1000 \mathrm{AU}$. The radiation exposure is also low, with median FUV flux $G_{0} \sim 900\left(1.4 \mathrm{erg} \mathrm{s}^{-1} \mathrm{~cm}^{-2}\right)$, so that photoevaporation of circumstellar disks is only important beyond $30 \mathrm{AU}$. Given the low interaction rates and modest radiation levels, we suggest that solar system disruption is a rare event in these clusters.
\end{abstract}

Subject headings: open clusters and associations: general - stars: formation - planets: formation 


\section{Introduction}

Current data indicates that a significant fraction of the stellar population is born in groups and clusters embedded within the densest regions of giant molecular clouds (GMCs). Advances in infrared astronomy during the past two decades have afforded astronomers with an unprecedented view of these stellar nurseries. These clouds form relatively rapidly (1-10 Myr) out of intergalactic gas and dust as a result of the complex (and poorly understood) interplay of spiral density waves, supernova explosions, phase transitions, and instabilities (e.g., Elmegreen 1991). Once formed, GMCs obtain a highly clumpy structure, possibly due to collisions in supersonic turbulent flows (e.g., Klessen, Heitsch \& Mac Low 2000). This highly nonuniform structure contains numerous cores with masses ranging from a few to a few thousand solar masses. These dense cores (which have been mapped in $\mathrm{NH}_{3}$ - see the compilation of Jijina et al. 1999) are the sites of star formation. Specifically, fragmentation within the more massive cores $\left(M>50 M_{\odot}\right)$, possibly resulting from Jeans instability, decoupling of fluid and MHD waves (Myers 1998), and/or from the decay of turbulence (Klessen \& Burkett 2000, 2001), form gravitationally unstable substructures whose subsequent collapse leads to the formation of protostars (e.g., Shu 1977; Fatuzzo et al. 2004). At the end of this complex process, young embedded groups/clusters appear to be basic units of star formation, accounting for a significant fraction (perhaps as high as 90\%) of the stars that populate our galactic disk. The evolution of these young clusters and their resulting effects on stellar and planetary formation represents a fundamental set of astrophysical problems.

The "typical" size of star formation aggregates remains poorly defined. Lada \& Lada (2003) and Porras et al. (2003) present catalogs of nearby embedded clusters, the former including systems with $N \geq 30$ out to $2 \mathrm{kpc}$, and the latter including systems with $N \geq 10$ out to $1 \mathrm{kpc}$. The cumulative distributions for the number of stars born in units of size $N$, as a function of $N$, are presented in Figure 1 for both catalogs. The open squares represent the $2 \mathrm{kpc}$ sample and the open triangles represent the $1 \mathrm{kpc}$ sample; the dashed curve shows the $1 \mathrm{kpc}$ sample subjected to the same criteria as the $2 \mathrm{kpc}$ sample $(N \geq 30)$. The two samples provide a consistent estimate for the probability distribution of group/cluster sizes. One should keep in mind that these samples are not complete. Some of the distant groups/clusters in the sample may have larger stellar membership (than reported) because the faint (low mass) end of the stellar IMF is not fully observed. On the other hand, small groups with $N \sim 30-100$ may well exist and not be included in the samples at all. As a result, the true distribution of cluster sizes $N$ could be skewed toward either higher or lower $N$ than shown in Figure 1. For the sake of definiteness, however, in this paper we take this sample to be representative.

Large clusters like the Trapezium in Orion (with $N>1000$ ) are known to be disruptive to the star formation process (e.g., Störzer \& Hollenbach 1999). In contrast, small groups with $N \leq 100$ often have relatively little impact (e.g., Adams \& Myers 2001). As shown in Figure 1, however, the majority of stars observed in embedded clusters are found in systems that contain between 100 and 1000 members (at least for these observational samples). The evolution of these intermediate-sized systems and their effects on star and planetary formation are thus of fundamental 
importance. These systems can influence star and planet formation through dynamical interactions among kernels, competitive accretion, scattering interactions among star-disk systems and/or or early planetary systems, and by disruptive radiation from other stars (especially the larger ones that live near cluster centers).

This paper considers the dynamics of intermediate-sized stellar systems with $N=100-1000$. In the two data sets described above (Lada \& Lada 2003; Porras et al. 2003), the fraction of stars that are found in systems with $N<100$ is $19 \%$ and $20 \%$, respectively, whereas the fraction of stars found in systems with $N>1000$ is $24 \%$ and $20 \%$. The majority of stars (about $60 \%$ ) are found in systems within our range of study. A large body of previous work on the dynamical evolution of $N$ body systems exists. The evolution of stellar clusters has been investigated for both small $N \leq 100$ (Lada, Margulis \& Deardorn 1984) and large $N>10,000$ (e.g., Portegies Zwart et al. 1998; Boily \& Kroupa 2003b). The dynamical effects of binaries has also been explored both in the context of globular clusters (Hut \& Bahcall 1983) and young clusters (Kroupa, Petr, \& McCaughrean 1999; Kroupa \& Bouvier 2003). Some work on intermediate sized systems has been performed (see Kroupa 1995b and references therein). On a smaller system scale, planetary disruption has been explored by numerous authors (e.g. de la Fuente Marcos \& de la Fuente Marcos 1997, 1999; Adams \& Laughlin 2001; Smith \& Bonnell 2001; Hurley \& Shara 2002; David et al. 2003; Fregeau, Chatterjee, \& Rasio 2005), and the few-body problem has been investigated by Sterzik \& Durisen (1998). Although these works have greatly advanced our understanding of the dynamics of manybody systems, a great deal of work remains to be done. This paper concentrates on the range of parameter space subtended by most young stellar clusters, $N$ in the range $100 \leq N \leq 1000$, and seeks to determine the effect of the cluster environment on forming stars and planetary systems.

In addition to its focus on intermediate sized clusters, this work differs from previous studies in the starting conditions. Most previous $N$-body simulations of stellar groups have invoked virial arguments to set the initial velocities of the system members. A distinguishing aspect of this study is the adoption of subvirial starting conditions. This initial condition is motivated by clump dispersion measurements obtained from recent observations of four systems in which the stars are (apparently) born with speeds substantially lower than virial (assuming that observed clumps are progenitors of individual protostars or stars). Specifically, in the NGC 1333 cluster, the observed clump-to-clump RMS velocity is only $\sim 0.45 \mathrm{~km} / \mathrm{s}$, significantly less than that expected if the clumps were in virialized orbits $(v \sim 1 \mathrm{~km} / \mathrm{s})$; furthermore, the clump-to-clump RMS velocity is much lower for subgroups within the larger complex (Walsh et al. 2004, Walsh, Myers, \& Burton 2004). Similarly, the velocity dispersion for 45 clumps (condensations) in $\rho$ Oph was estimated to be $\sim 0.64 \mathrm{~km} / \mathrm{s}$, with similar results obtained for 25 clumps in the NGC 2068 protocluster (see André 2002 and references therein). As another example, the clump to clump velocities in the NGC 2264 region are estimated to be about 3 times smaller than that expected in virial equilibrium (Peretto, André, \& Belloche 2005).

This paper undertakes a statistically comprehensive study of the dynamical evolution of young stellar clusters with populations in the range $100 \leq N \leq 1000$ and uses $N$-body simulations to follow 


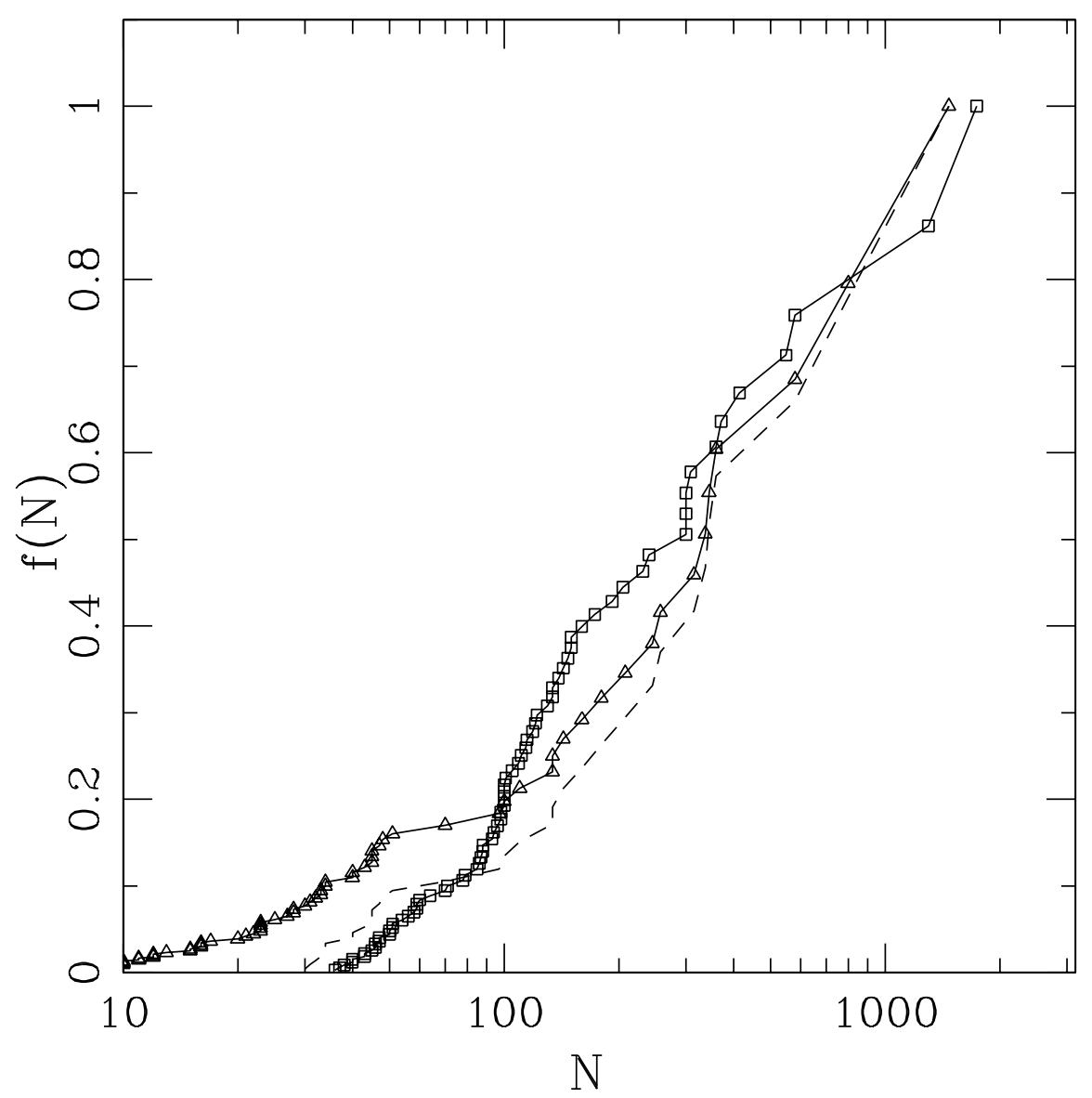

Fig. 1.- Cumulative distribution of group/cluster sizes as a function of system size $N$. The quantity $f(N)$ is the fraction of the total number of stars in the sample that live in groups/clusters of system size $N$ or smaller. The curve marked by open squares corresponds to the $2 \mathrm{kpc}$ sample, which is complete down to $N=30$ (Lada \& Lada 2003); the curve marked by open triangles is the $1 \mathrm{kpc}$ sample, which is complete down to $N=10$ (Porras et al. 2003). The dashed curve shows the $1 \mathrm{kpc}$ sample subjected to the same selection criteria as the $2 \mathrm{kpc}$ sample. 


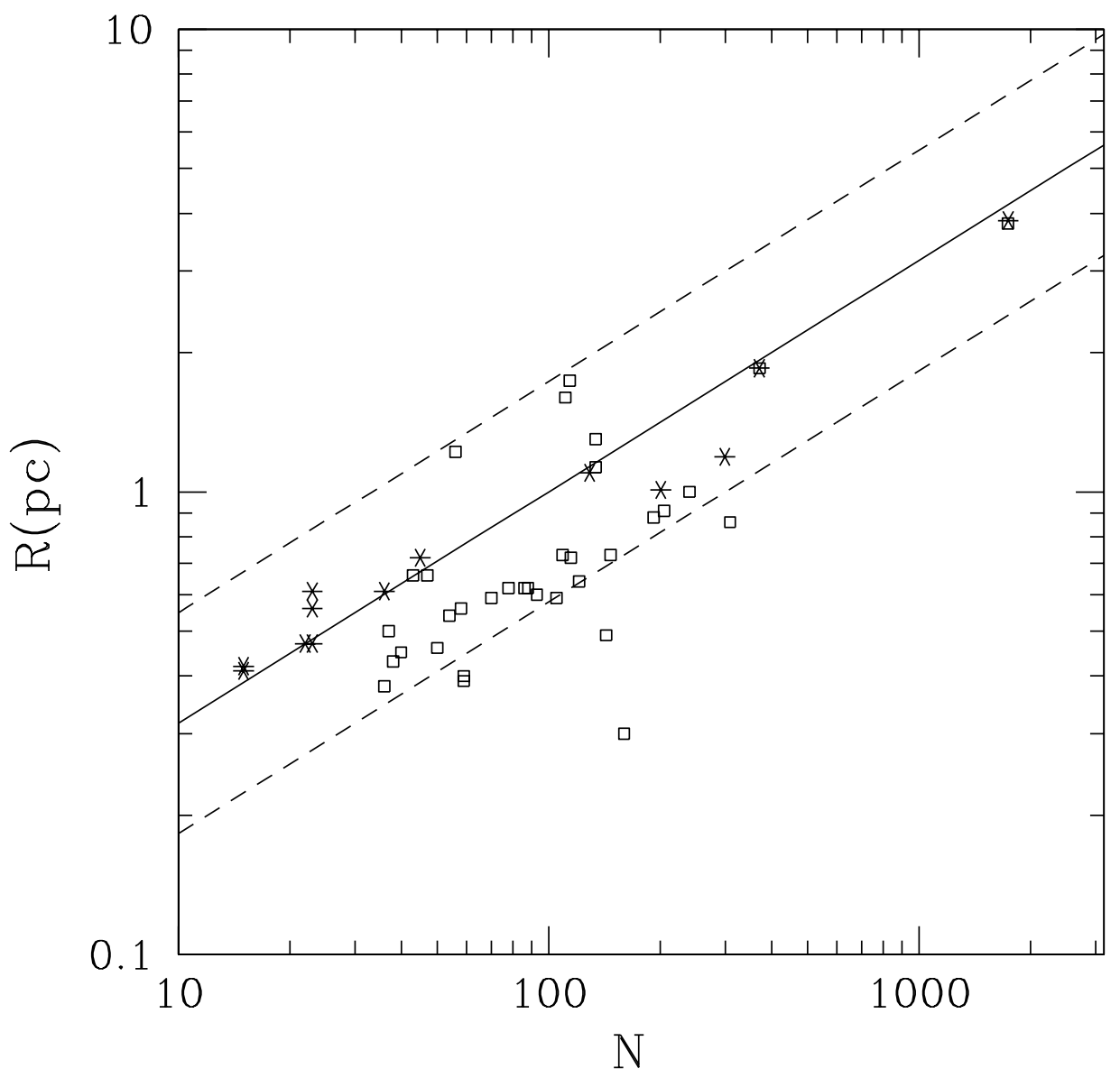

Fig. 2.- Observed group/cluster sizes as a function of system size $N$. The open squares represent data taken from the compilation of Lada \& Lada (2003); the stars represent data from the work of Carpenter (2000). The solid curve is a rough fit to the data with the form $R(N)=R_{300}(N / 300)^{1 / 2}$ with $R_{300}=\sqrt{3} \mathrm{pc}$; the two dashed curves have the same functional dependence with the length scale $R_{300}$ larger or smaller by a factor of $\sqrt{3}$. For most of this work we use the lower curve, with $R=1$ pc $(N / 300)^{1 / 2}$ in order to determine the greatest possible effects of the cluster environment. 
these systems from their nascent, embedded stages out to ages of $10 \mathrm{Myr}$. One goal of this study is to explore how early evolution depends upon the number $N$ of system members. The systems begin with a gaseous component which is subsequently removed (e.g., at time 5 Myr). Multiple realizations of equivalent initial conditions are performed in order to build up robust distributions of the output measures. We find that 100 realizations (simulations) of each set of initial conditions are required to provide good statistics for the output measures. These measures include closest approaches of cluster members, their radial locations and mass profiles (which largely determine the radiation exposure), as well as the time evolution of the bound cluster fraction, the virial ratio, the velocity isotropy parameter, and the half-mass radius. Because of the large number of simulations required for each set of initial conditions, we limit this preliminary study to six cluster types: $N=$ 100, 300, and 1000, with both "cold" (subvirial) and virial initial conditions.

The output measures are used to determine the impact of the cluster environment on star and planet formation. Toward that end, we determine the distribution of FUV luminosities for groups and clusters as a function of system size $N$. This ultraviolet radiation acts to destroy circumstellar disks and to inhibit planet formation. This work provides a measure of its efficacy as a function of group/cluster size $N(\S 3)$. We also calculate the cross sections for newborn planetary systems to be disrupted by passing stars (binaries). These cross sections ( $§ 4)$ are used in conjunction with the distributions of closest approaches from the $N$-body simulations to provide a measure of solar system disruption as a function of system size $N$. Armed with a robust statistical description of the evolution of young clusters, we undertake a detailed analysis of the particular system NGC 1333 $(\S 5)$. Recent observations of this young cluster (Walsh et al. 2005) provide position and velocity information on the $N=93 \mathrm{~N}_{2} \mathrm{H}^{+}$clumps found within the system. Since the observations specify only three of the six components of phase space, we must reconstruct the cluster conditions through multiple realizations, thereby producing an ensemble of calculations that can then be compared with the results of our theoretical study. Our results and conclusions are summarized in $\S 6$.

\section{Numerical Simulations of Young Embedded Clusters}

For the first part of this study we perform a suite of $N$-body simulations for intermediate-sized clusters as they evolve from their embedded stage out to ages of $\sim 10 \mathrm{Myr}$. Cluster evolution depends on the cluster size $N$, the initial stellar profile, the initial gas profile, the star formation history, the stellar IMF, and the gas disruption history. Given the large number of parameters needed to adequately describe young clusters (see also below), this initial study does not consider every combination of parameters that these systems could attain. Instead, we identify a baseline set of parameters that represent a "typical" cluster and perform many realizations of this benchmark model. We find that for every set of cluster parameters, one must perform many realizations of the initial conditions in order to fully sample the output measures. This study explores the variation of the cluster size $N$ and the effects of subvirial versus virial starting conditions. A forthcoming follow-up study will consider a much wider exploration of parameter space. For each set of input 
parameters, we perform 100 equivalent realizations in order to build up a statistical representation of the output measures. The input parameters and output measures are described below.

The $N$-body integrations are performed using NBODY2 (Aarseth 1999, 2001). This version of the integration package is relatively fast and allows for many realizations of each set of initial conditions to be run, as required to obtain good statistics. In this initial study, however, we do not include the binarity of the stellar systems. In sufficiently dense and long-lived clusters, binaries can absorb and store enough energy to affect the evolution of the cluster system. This paper focuses on the dynamics of systems with $N=100-1000$, where we expect interactions to be sufficiently rare and sufficiently distant that binarity has only a small effect on overall energy budget of the cluster (see also Kroupa 1995, Kroupa et al. 2003). This approximation is checked for consistency in two ways. First, we perform a test simulation including binaries (using NBODY6; Aarseth 1999) and find that the results are the same. As a second check, we use the distributions of closest approaches found from our ensemble of simulations and find that binary interactions are not energetically important in these systems (see below).

\subsection{Parameter Space}

Cluster membership $N$. Figure 1 indicates that most stars form in clusters with stellar membership $N$ in the range $100 \leq N \leq 1000$, with roughly half of stars belonging to clusters with size $N<300$ (and half with $N>300$ ). We thus consider the value $N=300$ as the center of our parameter space, and explore the evolution of clusters with $N=100,300$, and 1000 .

Initial cluster radius $R_{c *}$. Young clusters are found to have radii $R_{c *}$ within the range $0.1-2$ pc. An observationally determined relation between $R_{c *}$ and $N$ is shown in Figure 2, where open squares represent data taken from the compilation of Lada \& Lada (2003) and stars represent data from Carpenter (2000). A correlation between $R_{c *}$ and $N$ is clearly evident, although significant scatter exists. The data can be fit by the relation of the form

$$
R_{c *}(N)=R_{300} \sqrt{(N / 300)}
$$

where $R_{300} \approx 1-2$ pc. This relation corresponds to a nearly constant surface density of stars $N / R^{2} \approx$ constant. The solid curve shown in Figure 2 uses $R_{300}=1.7 \mathrm{pc}$; the dashed curves have

the same functional dependence but are scaled (up or down) by a factor of $\sqrt{3}$, and quantify the spread in this correlation. For this study we adopt this functional dependence to specify the initial radius of the stellar component and use $R_{300}=1.0 \mathrm{pc}$. This value is near the lower end of the observed range and thus maximizes the density, which in turn leads to dynamical interactions near the upper end of the range expected in these cluster systems.

Initial stellar profile. Embedded clusters display structure that can be characterized as centrally condensed or hierarchical (Lada \& Lada 2003). In a complete treatment, one should explore both spherical and nonspherical stellar distributions. In this initial study, however, we focus on 
the spherical case, where stars are randomly placed within a sphere of radius $R_{c *}$. For the sake of definiteness, the initial density of stars is taken to have the form $\rho_{*} \sim r^{-1}$ so that the initial stellar mass component is distributed according to $M_{*}(r) \sim r^{2}$ (out to the boundary at $R_{c *}$ ). This form is consistent with the expected density profiles for gas in cluster forming cores (see below).

Although there is evidence for a nearly universal initial mass function (IMF) for stars in young clusters, it remains unclear how stellar mass correlates with the initial position within a cluster. Massive stars are preferentially found near the centers of open clusters (e.g., Elmegreen et al. 2000), but the same trend need not be universally true for embedded clusters. Some clusters show evidence for mass segregation (Testi et al. 1998; Hillenbrand \& Hartmann 1998; Jiang et al. 2002) and theoretical considerations suggest that mass segregation has a primordial origin in some systems (Bonnell \& Davies 1998; Hillenbrand \& Hartmann 1998; see also Carpenter et al. 1997). However, the relative importance of dynamical versus primordial mass segregation in clusters with $100<N<1000$ remains uncertain. Given the evidence for some primordial mass segregation, we adopt a simple algorithm consistent with observed groups: For a given system, we sample the stellar masses from a standard IMF, and then relocate the most massive member to the cluster center. The remaining stars are then placed randomly so that the initial stellar component has density $\rho_{*} \sim r^{-1}$ within the radial range $0 \leq r \leq R_{c *}$. This approach thus provides a minimal treatment of primordial mass segregation. A more detailed treatment should be considered in follow-up studies. The issue of mass segregation is important because massive stars can produce powerful winds, outflows, and radiation fields that, if centralized, can more readily disrupt the gaseous component of a cluster (as well as planet forming disks around other stars).

Initial speeds. As discussed above, stars often appear to be born in young embedded clusters with initial speeds substantially less than the virial values (André 2002, Walsh et al. 2004, Peretto et al. 2005). To set the initial stellar velocities, we sample from a distribution that is characterized by a given expectation value for the virial ratio $Q \equiv|K / W|$ (Aarseth 2003), i.e., the ratio of kinetic to potential energy, where $Q=0.5$ for virialized systems. One goal of this study is to explore the effects of subvirial starting conditions. For the sake of definiteness, we adopt a baseline value of $Q=0.04$ for our subvirial simulations (i.e., starting speeds about $30 \%$ of the value needed for virial equilibrium). For comparison, we also study the virilized initial condition $Q=0.5$ for (otherwise) the same starting conditions. We refer to the subvirial starting states as "cold" and the virial initial states as "virial".

Spread in star formation times. A system of stars evolving from such an initially "cold" state would collapse into a dense core within a crossing time if all of the stars formed (and hence began falling toward the center) at exactly the same time. The resulting traffic jam at the cluster center would be unphysical, however, because the stars must have a spread in formation time. In this study, we assume that forming stars are tied to their kernels (the collapsing pockets of gas), which are moving subsonically, until the collapse phase of an individual star formation event is completed. After their collapse phase, newly formed stars are free to fall through the gravitational potential of the group/cluster system. Here we assume that the star formation epoch lasts for a given span of 
time $\Delta t=1 \mathrm{Myr}$, which is comparable to the crossing time. For comparison, the expected collapse time for an individual protostar is much smaller, only about 0.1 Myr (see Shu 1977; Adams \& Fatuzzo 1996; Myers \& Fuller 1993).

Initial gas potential. Observations of young embedded clusters indicate that the gas density profiles may have (roughly) the form $\rho \sim r^{-1}$ (Larson 1985, Myers \& Fuller 1993, Jijina et al. 1999; see also the discussion of McKee \& Tan 2003) on the radial scale of the cluster $(\sim 1 \mathrm{pc})$. For these simulations we need to include the graviational potential of the gaseous component and eventually let it disappear with time. In order to smoothly extend the initial gas potential out to large radii, we adopt a Hernquist profile so that the initial gas distribution is characterized by the potential, density, and mass profiles of the forms

$$
\Psi=\frac{2 \pi G \rho_{0} r_{s}^{2}}{1+\xi}, \quad \rho=\frac{\rho_{0}}{\xi(1+\xi)^{3}}, \quad \text { and } \quad M=\frac{M_{\infty} \xi^{2}}{(1+\xi)^{2}},
$$

where $\xi \equiv r / r_{s}$ and $r_{s}$ is a scale length (Hernquist 1990). Notice that $M_{\infty}=2 \pi r_{s}^{3} \rho_{0}$. In practice we identify the scale $r_{s}$ with the cluster size (Fig. 2), so that $r_{s}=R_{c *}$. The density profile within the cluster itself thus has the form $\rho \sim r^{-1}$; the steeper density dependence $\rho \sim r^{-4}$ occurs only at large radii (effectively outside the cluster) and allows the potential to smoothly join onto a force-free background. The mass enclosed within $\xi=1$, denoted here as $M_{1}$, is the effective gas mass within the cluster region itself (notice that the density and mass profiles extend out to spatial infinity and that the asymptotic mass $M_{\infty}=4 M_{1}$ ). The star formation efficiency (SFE) within the cluster is thus given by $\mathrm{SFE}=M_{*} /\left(M_{*}+M_{1}\right)$. Although observational determinations of SFE are subject to both uncertianties and system-to-system variations, typical values for a sample of nearby embedded clusters lie in the range $\mathrm{SFE}=0.1-0.3$ (Lada \& Lada 2003). This study adopts a baseline value $M_{1}=2 M_{*}$ (so that SFE $=0.33$ ). Thus, the mass that will end up in stars over the time interval $\Delta t=1 \mathrm{Myr}$ is pre-determined. Over the time $\Delta t$, the stellar masses become dynamically active and begin to fall through the potential (thus, the total mass of the cluster is kept constant over the time $\Delta t$ when stars are being formed).

Gas removal history. Stellar aggregates are initially deeply embedded in dense gas, but they quickly disrupt the gaseous component through the action of stellar winds and outflows, radiative processes, and supernovae (e.g., Whitworth 1979; Matzner \& McKee 2000; Gutermuth et al. 2004). Although the details of the gas removal processes are not fully understood, observations indicate that clusters older than about $5 \mathrm{Myr}$ are rarely associated with molecular gas, so that gas removal must occur in these systems on a comparable timescale (Lada \& Lada 2003). The fraction of stars that remain gravitationally bound after gas removal has been explored both analytically (e.g., Adams 2000; Boily \& Kroupa 2003a) and numerically (e.g., Lada, Margulis \& Dearborn 1984; Geyer \& Burkert 2001; Boily \& Kroupa 2003b). Gas affects the dynamical evolution through its contribution to the gravitational potential. As gas leaves the system, the gravitational well grows less deep and the stellar system adjusts its structure. Stars filling the high velocity part of the distribution will thus leave the system, but a fraction of stars can remain bound after the gas has been removed. The value of this fraction depends on the star formation efficiency, the geometries of 
the gaseous and stellar components, the gas dispersal history, and the stellar distribution function. This paper uses a simple model for gas removal: The gas is removed instantaneously at a given time $t=5$ Myr (e.g., Leisawitz, Bash, \& Thaddeus 1989) after the star formation process begins (recall that stars are randomly introduced over a time interval $\Delta t$, the beginning of which defines the time $t=0$ ). This choice of parameters allows the gas to remain in the system as long as possible (according to the currently available observations - see Lada \& Lada 2003). These simulations thus represent an upper limit on the level of interactions expected in astronomical clusters. Notice also that the gas potential is considered fixed while gas remains within the cluster. For clusters with cold starting conditions, the stars fall toward the cluster center and the gas could become more concentrated as well. This effect is small in the present case because gas dominates the potential, but could be considered in further work.

Binary test. In order to test the validity of our approximation of ignoring binarity, we performed a test simulation using both NBODY6 (which includes binaries - Aarseth 1999) and NBODY2 (where the masses of the two binary companions are combined to make a single star). The comparison runs are made for a cluster with $N=300$ and radius $R=1 \mathrm{pc}$, which defines the center of our parameter space (see above). We also use a cold start, an initial $Q=0.04$, because the cold runs should have more interactions and hence be more affected by binaries. In the test runs, gas is included as a Plummer sphere (with scale radius $R_{s}=1 \mathrm{pc}$ ) since the original $N$-body codes are written with the Plummer potential. The gas mass is equal to the total stellar mass. Over a time scale of $10 \mathrm{Myr}$, we find that the evolution of the fraction $f_{b}$ of bound stars, the virial parameter $Q$, and the half-mass radius $R_{1 / 2}$ are virtually identical for the two cases.

\subsection{Output Measures}

One goal of this work is to provide a statistical description of the systems under study. Two systems with identical sets of cluster parameters $\left(N, R_{c *}, \ldots\right)$ will have stars located at different starting locations and can evolve in different ways (for example, the history of close encounters will change). To provide a more complete description of the evolution of young clusters, we perform an ensemble of "effectively equivalent" simulations through multiple realizations of the system, i.e., we use the same set of cluster parameters but different choices for the random variables. In this manner, we can build up full distributions for the output measures of the systems (see also

Goodman Heggie, \& Hut 1993; Gierrsz \& Heggie 1994; Baumgardt, Hut, \& Heggie 2002; and references therein).

\subsubsection{Time evolution}

To characterize the time evolution, a variety of output measures are computed for each simulation, including the cluster's bound fraction, virial ratio, half-mass radius, and velocity isotropy 
parameter. These measures are calculated every 0.25 Myr throughout each 10 Myr simulation. For each system studied, the output measures of all the realizations (effectively equivalent simulations) are combined and averaged. We can then investigate the temporal evolution of each measure as well as use the measures to compare the different systems studied.

One important quantity is the fraction $f_{b}$ of stars that remain gravitationally bound as a function of time. For example, we would like to know how $f_{b}(t)$ depends upon the cluster size $N$ and the starting conditions (virial versus cold). The bound fraction $f_{b}$ of the cluster is defined by $f_{b} \equiv N_{\text {bound }} / N$, where $N$ is the initial number of stars in the cluster and $N_{\text {bound }}$ is the number of stars which have negative total energy at a given time. The bound fraction functions $f_{b}(t)$ are shown in Figure 3 for the six types of clusters considered here. Gas is removed at time $t=5 \mathrm{Myr}$, so the fraction of bound stars decreases after that epoch. Figure 3 shows that the cold clusters retain more of their stars for longer times.

In addition to $f_{b}$, we track the evolution of three other cluster diagnostics. The half-mass radius $R_{1 / 2}$ is defined to be the radius that encloses half of the stellar mass that is still gravitationally bound to the system. Over the long term, the half-mass radius $R_{1 / 2}$ is an increasing function of time, although it can decrease during the initial evolution of cold clusters. Within groups/clusters, young stars are often born with speeds much smaller than that required for virial equilibrium, but attain larger (virial) speeds as the system evolves. We can monitor this approach to equilibrium by tracking the evolution of the virial ratio $Q$ (the ratio of kinetic to potential energy for the stellar population). We also track the evolution of the isotropy parameter $\beta \equiv 1-v_{\theta}^{2} / v_{r}^{2}$, where $v_{\theta}$ and $v_{r}$ are the (averaged) $\hat{\theta}$ and $\hat{r}$ components of the velocity. The isotropy parameter provides a measure of the degree to which the cluster members have radial orbits. An isotropy parameter of $\beta=0$ corresponds to an isotropic velocity distribution, whereas $\beta=1$ corresponds to a cluster where the members are moving primarily in the radial direction.

The time evolution of the aformentioned cluster diagnostics are shown in Figure 3 for cluster sizes $N=100,300,1000$, and for both "cold" and "virial" starting conditions. As shown, the bound fraction is a slowly decreasing function of time, with a substantial jump at $t=5$ Myr when the gas is removed. The half mass radius $R_{1 / 2}$ remains nearly constant until gas removal at $t=5$ Myr, when it becomes an increasing function of time. The isotropy parameter $\beta$ is substantially radial $(0<\beta<1)$ over the entire evolution time for clusters with cold starting conditions, but shows a slight downward tendency at late times, indicating some evolution towards isotropy. For virial clusters, the parameter $\beta$ is close to zero (isotropic) for the first $5 \mathrm{Myr}$ of evolution, but develops a definite radial characteristic $(\beta \sim 0.5)$ for the second half of the time interval after the gas is removed. These same general trends are evident in the ensemble of results summarized by Table 1 below. For each cluster size $(N=100,300,1000)$ and each starting condition ("virial" or "cold") we have found the average values of the fraction $f_{b}$ of stars that remain bound after 10 Myr. Similarly, we have found averages of the viral parameter $Q$, the half-mass radius $R_{1 / 2}$, and the isotropy parameter $\beta$ for the first $5 \mathrm{Myr}$ (while the clusters retain gas) and the second $5 \mathrm{Myr}$ of evolution (when the clusters are gas-free). The final line of the table gives the output parameters 

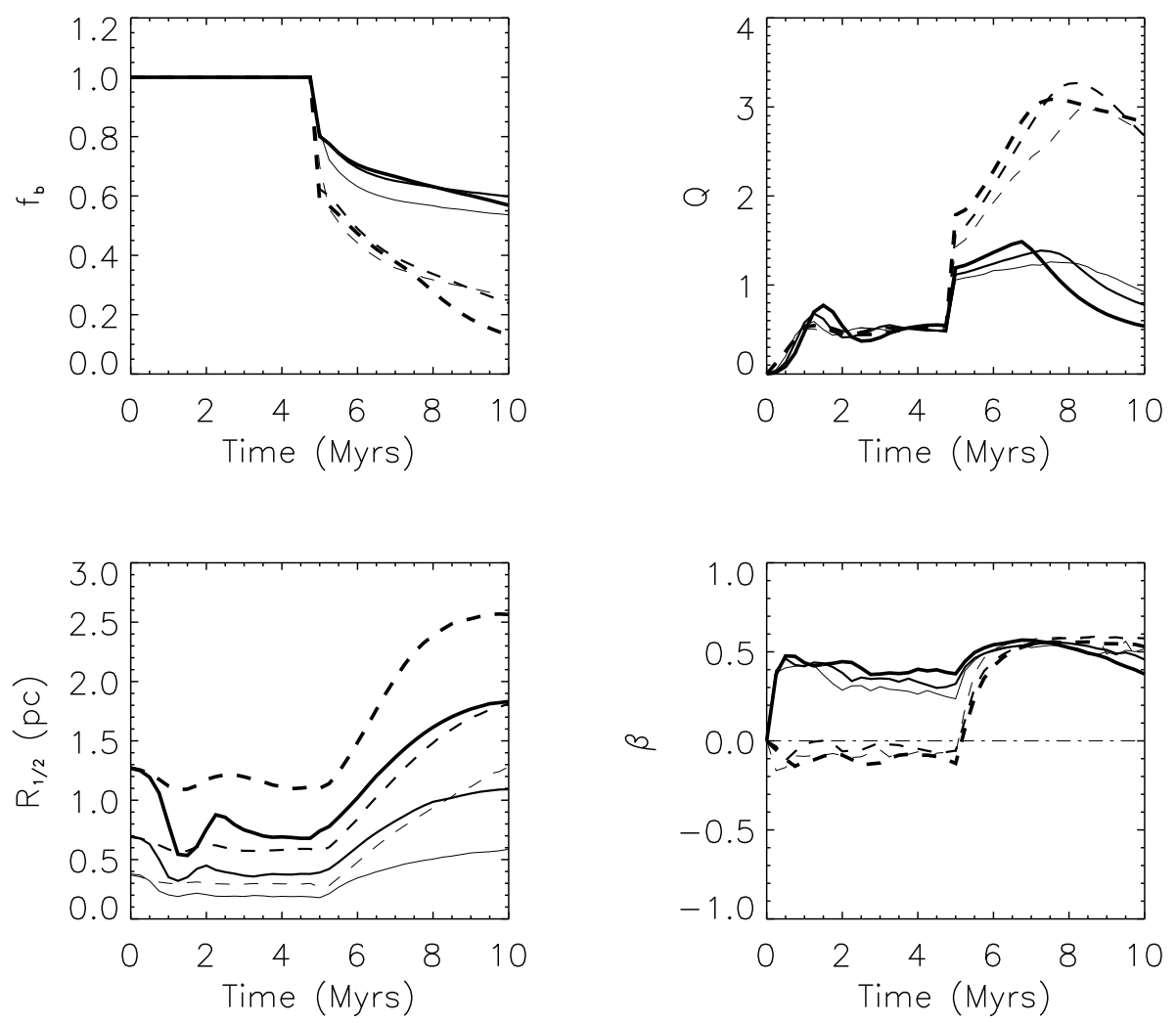

Fig. 3. - Time evolution for output measures for cluster simulations with $N=100,300$, and 1000. In each panel, the solid curves show the time evolution functions for "cold" initial conditions and the dashed curves show the time evolution for "virial" starting conditions. The boldness of the curves denotes the cluster size, with the darkest curves for $N=1000$ and the lightest curves for $N$ $=100$. The upper left panel shows the fraction of stars that are bound as a function of time, the upper right panel shows the time evolution of the virial ratio $Q \equiv|K / W|$, the lower left panel shows the time evolution of the half-mass radius, and the lower right panel shows the velocity isotropy parameter $\beta$. In all of these simulations, the gas is removed at time $t=5 \mathrm{Myr}$, which leads to structure in all of the time evolution functions as shown here. 
for our simulations of NGC 1333 (see $§ 5$ ).

Table 1: Cluster Evolution Parameters

\begin{tabular}{lccccccc}
\hline \hline & $f_{b}$ & $Q$ & $Q$ & $R_{1 / 2}(\mathrm{pc})$ & $R_{1 / 2}(\mathrm{pc})$ & $\beta$ & $\beta$ \\
Cluster Type & $10 \mathrm{Myr}$ & $0-5 \mathrm{Myr}$ & $5-10 \mathrm{Myr}$ & $0-5 \mathrm{Myr}$ & $5-10 \mathrm{Myr}$ & $0-5 \mathrm{Myr}$ & $5-10 \mathrm{Myr}$ \\
\hline 100 Cold & 0.536 & 0.489 & 1.15 & 0.211 & 0.457 & 0.320 & 0.502 \\
100 Virial & 0.265 & 0.511 & 2.48 & 0.301 & 0.832 & -0.0849 & 0.515 \\
300 Cold & 0.598 & 0.491 & 1.15 & 0.413 & 0.861 & 0.368 & 0.511 \\
300 Virial & 0.239 & 0.517 & 2.72 & 0.596 & 1.31 & -0.0404 & 0.513 \\
1000 Cold & 0.569 & 0.497 & 1.04 & 0.780 & 1.44 & 0.410 & 0.500 \\
1000 Virial & 0.130 & 0.527 & 2.74 & 1.15 & 2.09 & -0.0993 & 0.485 \\
\hline NGC 1333 & 0.689 & 0.525 & 0.690 & 0.117 & 0.238 & 0.230 & 0.339 \\
\hline \hline
\end{tabular}

\subsubsection{Radial distributions}

As a group/cluster system evolves, interactions between members result in a distribution of stellar positions and velocities. As the gas is removed from the system, high-velocity stars are more likely to become gravitationally unbound and leave the system, whereas low velocity stars tend to condense into a central bound core. Complicating this process, dynamical mass segregation also takes place, albeit on somewhat longer time scales. As one way to characterize the evolution of these sytems, we produce mass profiles $M(r)$ averaged over the $10 \mathrm{Myr}$ time interval of interest. Specifically, the radial position of every star is recorded at intervals of $0.25 \mathrm{Myr}$ throughout each simulation. The resulting data set is used to create a mass profile $M(r) / M_{T *}$ at each time, where $M_{T *}$ is the total mass in stars that remain bound. The profiles are then averaged over all time steps and averaged over the 100 equivalent realizations of the system to produce the radial mass profile associated with each type of group/cluster. The integrated mass distribution $M(r)$ can be fit with a simple function of the form

$$
\frac{M(\xi)}{M_{T *}}=\left(\frac{\xi^{a}}{1+\xi^{a}}\right)^{p}
$$

where $\xi=r / r_{0}$, and where the scale length $r_{0}$ and the index $p$ are free parameters that are fit to the output of the simulations. The index $a$ can also be varied: We find that the cold clusters can be fit with $a=2$, whereas the virial clusters require $a=3$. The best fit parameters for the various simulations are given in Table 2 below. The table also shows the fitting parameters for the mass profiles averaged over the first $5 \mathrm{Myr}$ (before gas removal) and over the second $5 \mathrm{Myr}$ (after gas has left the system). The final line of the table gives the fitting parameters for the simulations of NGC 1333 (see $\S 5$ ). Figure 4 shows the radial mass profiles from both the simulations and the fitting functions. The simulation profiles are time averaged over the first $10 \mathrm{Myr}$ of evolution (and over 100 realizations of each starting condition). 
Table 2: Output Parameters for Mass Distributions

\begin{tabular}{l|cc|cc|cc|l}
\hline \hline & \multicolumn{2}{|c|}{$(0-10 \mathrm{Myr})$} & \multicolumn{2}{|c|}{$(0-5 \mathrm{Myr})$} & \multicolumn{2}{|c|}{$(5-10 \mathrm{Myr})$} & \\
Cluster Type & $p$ & $r_{0}(\mathrm{pc})$ & $p$ & $r_{0}(\mathrm{pc})$ & $p$ & $r_{0}(\mathrm{pc})$ & $a$ \\
\hline 100 Cold & 0.686 & 0.394 & 0.680 & 0.264 & 1.02 & 0.453 & 2 \\
100 Virial & 0.436 & 0.698 & 0.406 & 0.486 & 0.776 & 0.793 & 3 \\
300 Cold & 0.785 & 0.635 & 0.747 & 0.484 & 1.01 & 0.781 & 2 \\
300 Virial & 0.493 & 1.19 & 0.489 & 0.846 & 0.605 & 1.56 & 3 \\
1000 Cold & 0.820 & 1.11 & 0.769 & 0.899 & 0.970 & 1.34 & 2 \\
1000 Virial & 0.586 & 1.96 & 0.590 & 1.53 & 0.689 & 2.45 & 3 \\
\hline NGC 1333 & 0.552 & 0.300 & 0.436 & 0.241 & 0.924 & 0.299 & 2 \\
\hline \hline
\end{tabular}

We can also find profiles $N(r)$ for the number of stars enclosed within the radius $r$. These profiles are essentially the probability distributions for the radial positions of the stellar members. These profiles $N(\xi) / N_{T}$ can be fit with the same form as equation (3). Although not shown here, the fitting parameters are nearly the same as those of the mass profiles and are used (in §3) when we need to calculate the probability of finding a star at radius $r$.

One goal of this study is to characterize this class of groups and clusters. Toward this end, recent observational studies have determined the central densities for clusters (e.g., Gutermuth et al. 2005). However, the mass profiles found here imply that the central denssity of these clusters suffers from an ambiguity: If a mass profile has the form given by equation (3), the density profile takes the form $\rho \propto a p \xi^{a p-3}\left(1+\xi^{a}\right)^{-(p+1)}$, which diverges in the limit $\xi \rightarrow 0$. As a result, the central density for these mass profiles - and this class of systems - is not well-defined. In contrast, the total depth of the gravitational potential is well-defined and can be written in the form

$$
\Psi_{*}=\frac{G M_{T *}}{r_{0}} \psi_{0} \quad \text { where } \quad \psi_{0} \equiv \int_{0}^{\infty}\left(\frac{1}{1+u^{a}}\right)^{p}
$$

where we assume that the mass profile has the form of equation (3), which defines the indices $a$ and $p$, as well as the scale length $r_{0}$. The total mass of stars in the cluster is $M_{T *}$, so that $\Psi_{*}$ represents the total depth of the stellar contribution to the potential. For embedded clusters, the gas contribution to the potential (eq. [2]) should be added to obtain the total potential. Notice that in the limit $a p \rightarrow 1$, the integral in the definition of $\psi_{0}$ diverges and the central potential is no longer defined. However, all of the clusters considered here display well-defined central potentials. For the indices listed in Table 2, the value of the dimensionless potential $\psi_{0}$ lies in the range $\psi_{0}$ $=1.5-5.4$. The resulting stellar potential can be written in terms of a velocity scale $\sqrt{\Psi_{*}}$, which falls in the range $\sqrt{\Psi_{*}}=0.64-2.4 \mathrm{~km} / \mathrm{s}$ for the clusters considered here. 


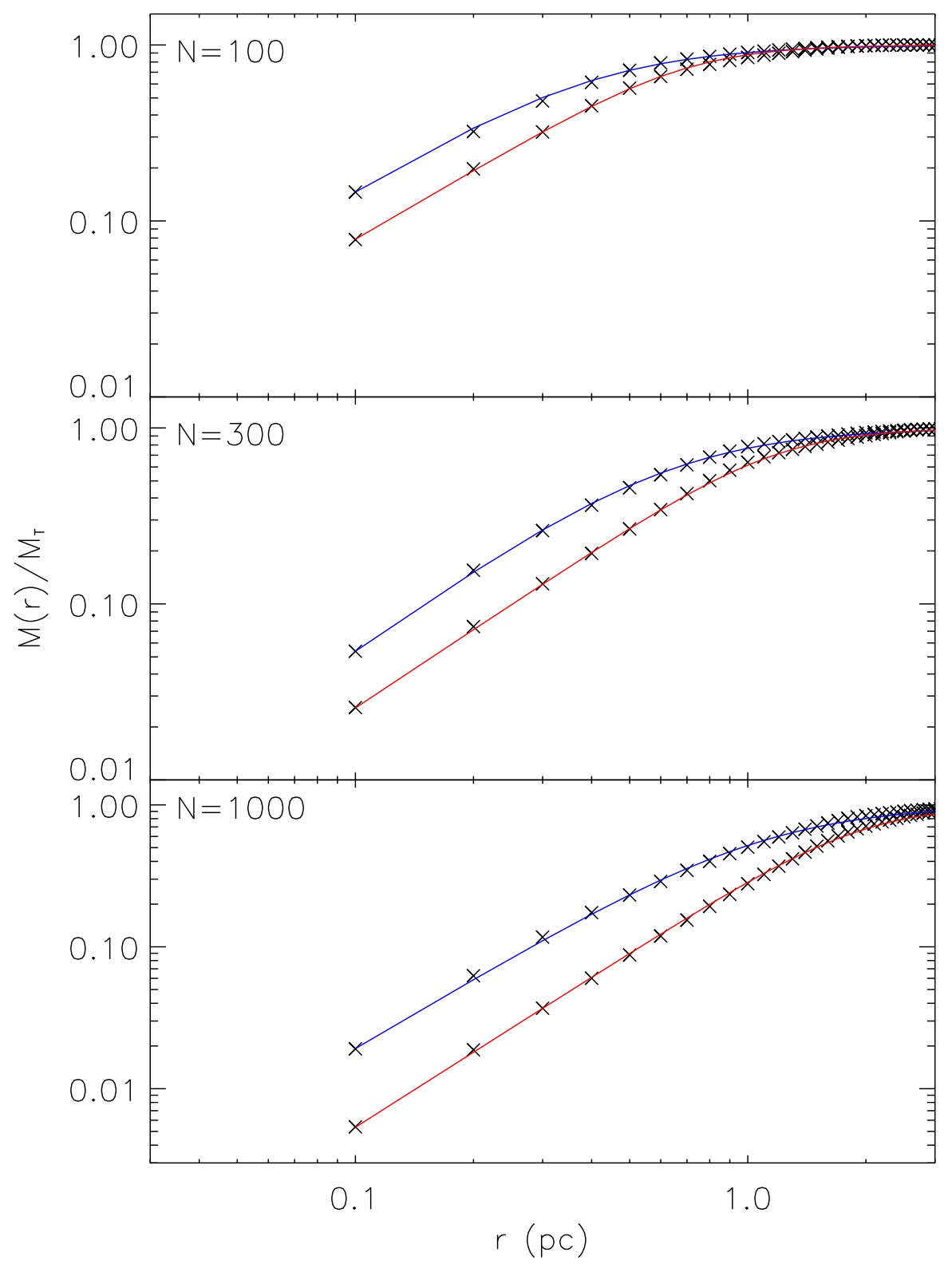

Fig. 4.- Time-averaged mass profiles $M(r) / M_{T *}$ for the six classes of starting conditions. The averages are taken over the first $10 \mathrm{Myr}$ and include only the stars that remain bound to the cluster (at each time). The top panel shows the stellar mass distribution $M(r)$ as a function of radius $r$ for clusters with $N=100$ and both "virial" (lower curve) and "cold" (upper curve) starting conditions. Each mass profile is compiled from the results of 100 simulations with different realizations of the same starting conditions. Similarly, the middle panel shows the mass distributions for clusters with $N=300$ and the bottom panel shows the distributions for $N=1000$. 


\subsubsection{Distribution of closest approaches}

The cluster environment facilitates close stellar encounters which can disrupt solar systems. Within the ensemble of $N$-body simulations described above, we can find the distributions of close encounters. These distributions, in conjunction with the cross-sections for disruptions of planetary systems (see §4; Adams \& Laughlin 2001), binary-disk systems (Ostriker 1994; Heller 1993, 1995; Kobayashi \& Ida 2001), and binary-star interactions (Heggie et al. 1996; McMillan \& Hut 1996; Rasio et al. 1995), can then be used to estimate the probability of interactions as a function of system size $N$ (and other initial conditions).

Specifically, the close encounters for each star are tracked throughout each cluster simulation; the resulting data is labeled with both stellar mass and cluster age. The total distribution of closest approaches for each simulation is calculated, and these distributions are then averaged over the 100 equivalent realizations of the system. The result is an integrated distribution of closest approaches for each type of cluster. The results are presented in terms of an interaction rate, i.e., the number of close encounters with $r \leq b$ that the "typical star" experiences per million years (1 Myr is a convenient unit of time and is approximately the cluster crossing time). This interaction rate is a function of closest approach distance $b$ and can be fit with an expression of the form

$$
\Gamma=\Gamma_{0}\left(\frac{b}{1000 \mathrm{AU}}\right)^{\gamma}
$$

The rate $\Gamma$ is thus the number of close encounters with $r \leq b$ per star per million years. For each type of group/cluster, the parameters $\Gamma_{0}$ and $\gamma$ were varied to find the best fits using the Levenberg-Marquardt procedure. The resulting parameter values are given in Table 3 for the six classes of systems studied here. The table also lists the fitting parameters for the closest approach distributions taken over the first 5 Myr of the simulations (when gas is still present) and the second 5 Myr time interval (after gas removal). These results are consistent with those obtained previously (e.g., Scally \& Clarke 2001 find similar interaction rates for the Orion Nebula Cluster, which is somewhat larger with $N=4000$ stars). Notice that the interaction rates are higher for the first 5 Myr interval than the second $5 \mathrm{Myr}$, by a factor of $\sim 5$, consistent with the spreading out of the cluster with time, especially after gas is removed at the $5 \mathrm{Myr}$ mark. Notice also that the total interaction rate over $10 \mathrm{Myr}$ is the average of the values over the two separate time intervals. The interaction rates are higher for the clusters with "cold" starting conditions. In these systems, the orbits are more radial than in the case of "virial" initial conditions (where the velocity distributions are more isotropic - see Table 1) and more of the stars pass near the cluster center where the density is higher.

The fitting functions (given by equation [5] and Table 3) provide a good working description of the distribution of closest approaches for each ensemble of simulations with given starting conditions. In order to interpret the meaning of these results, it is useful to compare with analytic estimates (Binney \& Tremaine 1987, hereafter BT87). For a cluster of size $N$ and radius $R$, the surface density of stars is roughly $N /\left(\pi R^{2}\right)$. For each crossing time $\tau_{c}$ of the cluster, a given star 
will thus experience close encounters at the rate

$$
\delta \Gamma_{a} \approx \frac{N}{\pi R^{2}} 2 \pi b \delta b \tau_{c}^{-1}
$$

with impact parameter (the closest approach distance in this approximation) between $b$ and $b+\delta b$ (BT87), where the time unit is the crossing time. The total rate $\Gamma_{a}$ of close approaches (at distance $\leq b)$ per crossing time is thus approximately given by

$$
\Gamma_{a} \approx \frac{N b^{2}}{R^{2}} \tau_{c}^{-1} \approx 0.007\left(\frac{b}{1000 \mathrm{AU}}\right)^{2} \tau_{c}^{-1}
$$

where we have used the observed scaling of cluster radius with $N$ (Figure 2 and equation [1]) to obtain the second approximate equality. Since the cluster crossing times $\tau_{c}$ are of order $1 \mathrm{Myr}$, this calculation produces an interaction rate with the same form as the fitting formula with $\Gamma_{0} \approx$ 0.007 and $\gamma=2$. As shown in Table 3, the fitting parameters for close approaches are in rough agreement with these estimated values, at the crudest level of comparison. The detailed forms are somewhat different - the numerically determined interaction rates are less steep (as a function of b) and somewhat larger than the analytic estimate.

The differences between the numerical interaction rates and the analytic estimate arise for several reasons. The crossing time is somewhat shorter than $1 \mathrm{Myr}$, so the rate increases accordingly. In addition, clusters have enhanced density in their centers and support more interactions there. Suppose that a cluster has a core, a long-lived central region of enhanced stellar density. If the core contains $N / 10$ stars at a given time and has radius $R_{c *} / 10$, the effective surface density, and hence the interaction rate per crossing time, would be 10 times higher than the estimate given above. Notice that the local crossing time would be smaller by a factor of $\sim 10$, but that stars would (on average) spend only $10 \%$ of their time in the core, so these latter two effects tend to cancel. The shallower slope of the distribution $(\gamma<2)$ is expected for sufficiently close encounters where the interaction energy $2 \mathrm{Gm} / \mathrm{b}$ is comparable to the typical velocity $V_{0}$ of a cluster star. In this case, the stars no longer travel on straight-line trajectories during the encounter (as implicitly assumed above) so that the impact parameter is larger than the distance of closest approach (BT87). Since $V_{0} \sim 1 \mathrm{~km} / \mathrm{s}$, this effect comes into play when $b \sim 2 G m / V_{0}^{2} \sim 900$ AU, i.e., just inside the regime of interest. In the extreme limit of small $b$, the interaction rate becomes linear $\Gamma_{a} \propto b\left(2 G m / V_{0}^{2}\right)$ so that $\gamma \rightarrow 1$. Notice that the slopes found from the numerical simulations lie in the range $1 \leq \gamma \leq 2$. 
Table 3: Output Parameters for Distributions of Closest Approach

\begin{tabular}{l|cc|cc|cc|c}
\hline \hline & \multicolumn{2}{|c|}{$(0-10 \mathrm{Myr})$} & \multicolumn{2}{|c|}{$(0-5 \mathrm{Myr})$} & \multicolumn{2}{c|}{$(5-10 \mathrm{Myr})$} & \multirow{2}{*}{ Cluster Type } \\
& $\Gamma_{0}$ & $\gamma$ & $\Gamma_{0}$ & $\gamma$ & $\Gamma_{0}$ & $\gamma$ & $b_{C}(\mathrm{AU})$ \\
\hline 100 Cold & 0.166 & 1.50 & 0.266 & 1.54 & 0.0672 & 1.42 & 713 \\
100 Virial & 0.0598 & 1.43 & 0.0870 & 1.46 & 0.0333 & 1.37 & 1430 \\
300 Cold & 0.0957 & 1.71 & 0.168 & 1.73 & 0.0240 & 1.59 & 1030 \\
300 Virial & 0.0256 & 1.63 & 0.0440 & 1.64 & 0.00700 & 1.53 & 2310 \\
1000 Cold & 0.0724 & 1.88 & 0.133 & 1.89 & 0.0112 & 1.83 & 1190 \\
1000 Virial & 0.0101 & 1.77 & 0.0181 & 1.79 & 0.00210 & 1.74 & 3650 \\
\hline NGC 1333 & 0.941 & 1.56 & 1.39 & 1.62 & 0.490 & 1.42 & 238 \\
\hline \hline
\end{tabular}

These simulations were performed using an $N$-body code that does not consider the binarity of the interacting units (NBODY2; Aarseth 1999). Given the distribution of closest approaches calculated here, we can check this approximation for self-consistency. For an interaction rate of the form of equation (5), and for a 10 Myr time span, the "typical" star will experience (on average) one encounter with the characteristic impact parameter $b_{C}$ given by

$$
b_{C} \equiv 1000 \mathrm{AU}\left(10 \Gamma_{0}\right)^{-1 / \gamma} .
$$

For the six classes of clusters considered here, the characteristic impact parameter lies in the range $b_{C}=700-4000 \mathrm{AU}$ (as listed in Table 3). For comparison, the peak of the binary period distribution is at $P \approx 10^{5} \mathrm{~d}$ (Duquennoy \& Mayor 1991), which corresponds to a separation of $\sim$ $42 \mathrm{AU} \ll b_{C}$. These results indicate that for the majority of binary systems, the separation is much less than the typically expected close approach $b_{C}$ (over the $10 \mathrm{Myr}$ time span considered here). Furthermore, the orbital energy at the typical flyby radius of a couple thousand AU corresponds to a velocity scale of $\sim 0.5 \mathrm{~km} / \mathrm{s}$, i.e., a typical star is expected to receive only a single velocity perturbation of this magnitude. Although this velocity kick is comparable to the mean velocity scale of the cluster given by $v_{m} \sim G N\langle m\rangle / R$, most of the interactions take place near the center of the cluster potential where $v \sim \sqrt{\Psi_{0}} \sim 3.5 v_{m}$, so the expected velocity perturbations are not devastating (one $\sim 15 \%$ kick in velocity, a $\sim 2 \%$ kick in energy). These results, taken together, imply that our approximation of ignoring binarity is justified. For completeness we note that some binary systems can be produced via three-body interactions during the evolution of a cluster. Care must be taken to identify these systems once formed, and to exclude the binary orbits from the determination of the closest approach distribution.

\subsubsection{Comparison of virial and cold starting conditions}

One issue of interest is the differences between the clusters with initial conditions where members are in virial equilibrium, $Q_{0}=0.5$ ("virial" clusters), and those where the members are started 


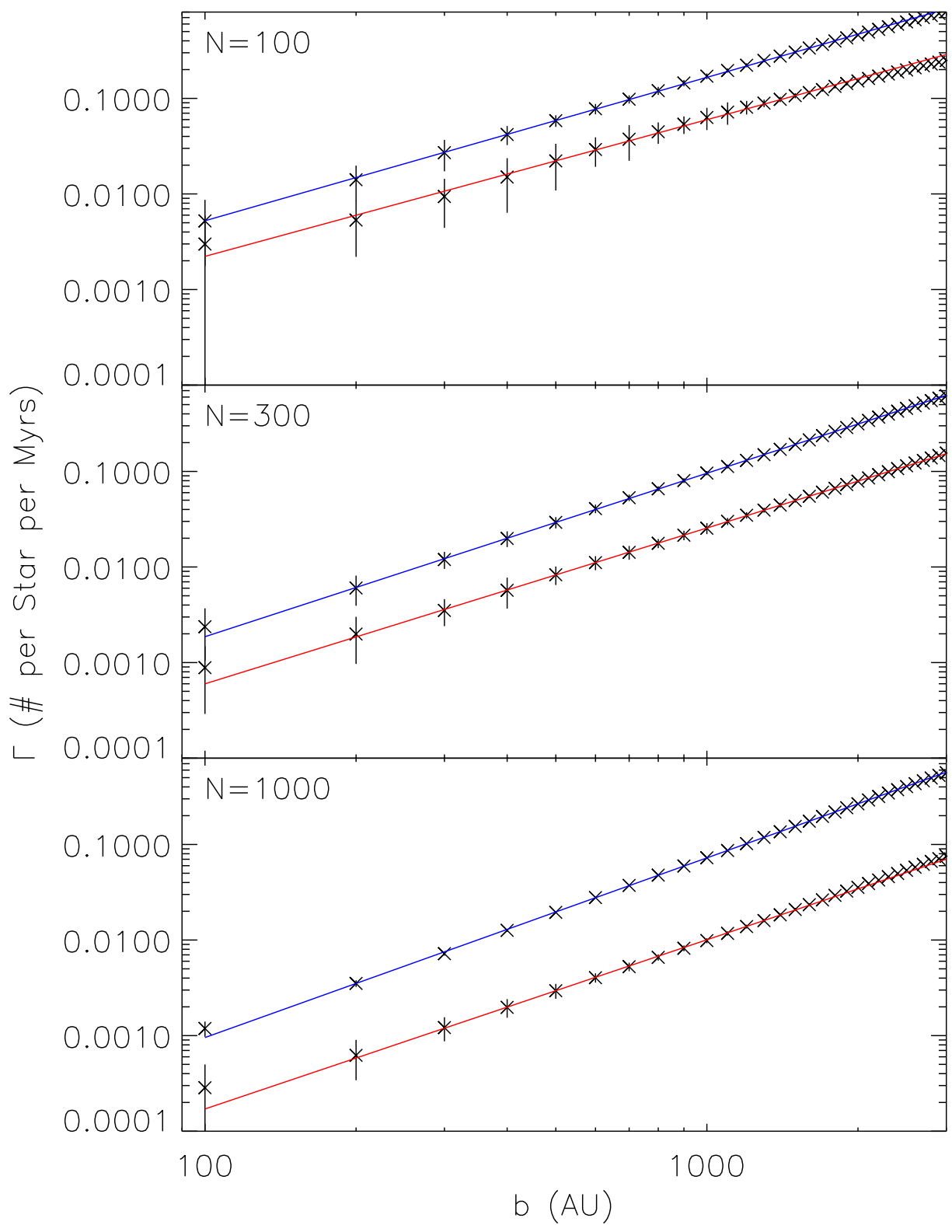

Fig. 5.- Distribution of closest approaches for the six classes of starting conditions. The top panel shows the distributions of closest approaches, plotted as a function of impact parameter, for clusters with $N=100$ and both "virial" (bottom curve) and "cold" (top curve) starting conditions. Each distribution is compiled from the results of 100 simulations, i.e., 100 realizations of the same starting conditions. Similarly, the middle panel shows the distributions of closest approaches for clusters with $N=300$ and the bottom panel shows the distributions for $N=1000$. The error bars shown represent the standard deviation over the compilations. 
with sub-virial velocities, $Q_{0}=0.04$ ("cold" clusters). The initial conditions lead to some important differences, as illustrated in Table 4, which lists the ratios of the parameters for the cold simulations to those from the virial simulations. The cold clusters have higher bound fractions, with $50-60 \%$ of their members remaining bound at $t=10 \mathrm{Myr}$. For comparison, the virial clusters have only $13-27 \%$ of the initial cluster membership bound at $t=10 \mathrm{Myr}$. In the cold clusters, stars fall toward the center after they form and thus spend more of their time inside the cluster gas (which is assumed to be static, i.e., not in a state of collapse). The removal of gas thus has less effect on the cold clusters and more stars remain bound. For both virial and cold clusters, the initial velocity distribution of the cluster members is isotropic. Since stars in the cold clusters are started with small velocities, however, they tend to fall toward the cluster center and rapidly develop relatively larger radial velocities. As a result, the isotropy parameter $\beta$ for cold clusters is larger (more radial with $\beta \approx 0.3-0.4$ ) than that of the virial clusters, which have $\beta \approx 0$ (and slightly negative) for the first $5 \mathrm{Myr}$ of evolution. After the gas is removed, both the virial and cold clusters have larger isotropy parameters, indicating increased radial motion as the cluster expands in the absence of the gas potential.

Table 4: Ratio of Parameters for Cold and Virial Initial Conditions

\begin{tabular}{cccccc}
\hline \hline $\begin{array}{c}N \\
(t=0)\end{array}$ & $f_{b}$ & $Q$ & $R_{1 / 2}$ & $R_{1 / 2}$ & $\Gamma_{0}$ \\
$0-10 \mathrm{Myr}$ & $5-10 \mathrm{Myr}$ & $0-5 \mathrm{Myr}$ & $5-10 \mathrm{Myr}$ & $0-10 \mathrm{Myr}$ \\
\hline 100 Stars & 2.02 & 0.464 & 0.701 & 0.549 & 2.78 \\
300 Stars & 2.50 & 0.423 & 0.693 & 0.657 & 3.74 \\
1000 Stars & 4.38 & 0.380 & 0.678 & 0.689 & 7.17 \\
\hline \hline
\end{tabular}

The mass distributions (Fig. 4) and distributions of closest approaches (Fig. 5) also depend on the starting conditions. The cold clusters have more centrally condensed radial profiles throughout the 10 Myr evolution time. Before gas expulsion, the half-mass radii for the cold clusters are about $70 \%$ of the half-mass radii of the virial clusters. This result can be understood if the cold clusters act as if they have zero temperature starting states, so they collapse to a radial scale roughly $\sqrt{2}$ times smaller than their initial size. In comparison, virial clusters tend to retain their starting radial size (before gas removal). After gas expulsion, the cold clusters continue to have half mass radii about $70 \%$ of those of the virial clusters, although the cold clusters with $N=100$ are somewhat more concentrated. The mass distributions show that the scale radii of the cold clusters are $55-65 \%$ of those of the virial clusters, and the central potentials are deeper by a factor of $1.2-2$. The distributions of closest approach for clusters with virial and cold starting conditions are similar and can be fit with a single power-law form over the radial range of interest (100-1000 AU). The power-law indices are roughly the same, but the cold clusters show a higher interaction rate by a factor of $3-7$. 


\section{Effects of Cluster Radiation on Forming Solar Systems}

Given the characterization of cluster dynamics found in the previous section, we now estimate the effects of ultraviolet radiation from the background cluster on nascent solar systems. The radiation from the parent star can drive mass loss from its planet-forming disk and thereby affect planet formation (Shu, Johnstone, \& Hollenbach 1993), but this effect is often dominated by radiation from the background star cluster (Johnstone, Hollenbach, \& Bally 1998; Adams \& Myers 2001). In this paper we focus on the effects of FUV radiation (Adams et al. 2004), which tends to dominate the effects of EUV radiation (Armitage 2000). We first calculate the distributions of FUV luminosity (§3.1) and then determine the extent to which the radiation compromises planet formation (§3.2). Throughout this section, we present results as a function of cluster size $N$. In this context, we consider the cluster to have a stellar membership of $N$ primaries and we ignore binarity. Although some fraction of the cluster members will have binary companions, the vast majority of the companions will have low mass and will not contribute appreciably to the FUV luminosity.

Notice that in the early stages of cluster evolution, the system will contain a substantial amout of gas and dust, and this dust can attenuate the FUV radiation. The gas (and dust) is removed from the cluster relatively early so that the FUV radiation eventually has a clear line of sight to affect forming planetary systems. Nonetheless, since we do not model the dust attenuation, the results of this section represent an upper limit to the effects of radiation.

\subsection{Probability Distributions for FUV Luminosity}

In this subsection, we calculate probability distributions for the FUV radiation emitted by stellar aggregates with varing size $N$ (the number of cluster members). The total FUV luminosity from a cluster or group of stars is given by the sum

$$
L_{F U V}(N)=\sum_{j=1}^{N} L_{F U V j},
$$

where $L_{F U V j}$ is the FUV luminosity from the $j t h$ member. In this approximation, we assume that the FUV luminosity for a given star is determined solely by the stellar mass. We further assume that the stellar mass is drawn from a known probability distribution, i.e., a known stellar initial mass function.

In this problem, low mass stars have a negligible contribution to the total UV flux. To a good approximation, we can ignore the contribution of all stars smaller than $1 M_{\odot}$. To specify the initial mass function, we are thus not concerned with the detailed shape at low stellar masses; we only need to correctly account for the fraction of stars with $M_{*}>1 M_{\odot}$ and the slope at high stellar masses. For the sake of definiteness, we assume that the stellar IMF has a power-law form for mass $M_{*}>1 M_{\odot}$ with index $\Gamma$, i.e.,

$$
\frac{d N}{d m}=A m^{-\Gamma}=\mathcal{F}_{1}(\Gamma-1) m^{-\Gamma}
$$


where $m$ is the mass in units of solar masses and where the slope $\Gamma=2.35$ for the classic form first suggested by Salpeter (1955); this slope remains valid today for a wide variety of regions (Massey 2003 and references therein). In the second equality, we have normalized the distribution according to the convention

$$
\int_{1}^{\infty} \frac{d N}{d m} d m=\mathcal{F}_{1}
$$

where $\mathcal{F}_{1}$ is the fraction of the stellar population with mass larger than $1 M_{\odot}$. For a typical stellar mass function (such as that advocated by Adams \& Fatuzzo 1996), the fraction $\mathcal{F}_{1} \approx 0.12$. For completeness, note that in practice we cut off the IMF at $m=100$ (see below); taking the integral out to $\infty$ here results in an inaccuracy of $\sim 0.2 \%$ (which is much smaller than the accuracy to which we know $\mathcal{F}_{1}$ ).

To specify the FUV luminosity as a function of stellar mass, $L_{F U V}(m)$, we use the models of Maeder and collaborators (see Maeder \& Meynet 1987; Schaller et al. 1992). Specifically, these papers provide a grid of stellar models as a function of both mass and age. We use the zero age models to specify the stellar luminosity and effective temperature. The FUV radiation is dominated by the largest stars, which reach the main-sequence rapidly. Since the model grids do not extend to arbitrarily high masses, we enforce a cutoff of $100 M_{\odot}$. Using a blackbody form for the atmosphere, we then calculate the fraction of the luminosity that is emitted in the FUV regime $(6 \mathrm{eV}<h \nu<$ $13.6 \mathrm{eV})$. The result is shown in Figure 6.

The expectation value $\left\langle L_{F U V}\right\rangle_{*}$ of the FUV luminosity is thus given by the integral

$$
\left\langle L_{F U V}\right\rangle_{*}=\int_{1}^{\infty} L_{F U V}(m) \frac{d N}{d m} d m \approx 8.20 \times 10^{35} \mathrm{erg} / \mathrm{s}
$$

This expectation value is normalized so that it is the expected FUV luminosity per star. Due to the wide range of possible stellar masses and the sensitive dependence of FUV emission on stellar mass, this expectation value is much larger than the FUV radiation emitted by the majority of stars. We thus only expect the FUV radiation from a given cluster to converge to that implied by the expectation value in the limit of larger numbers $N$ of stellar members, where large $N$ will be determined below. Small clusters will often experience large departures from the expectation value.

We want to calculate both the expectation value and its variance for the FUV luminosities of the entire cluster. The sum given by equation (9) is thus the sum of random variables, where the variables (individual contributions to the FUV power) are drawn from a known distribution (determined by the stellar IMF and the $L-m$ relation). In the limit of large $N$, the expectation value of the FUV power is thus given by

$$
L_{F U V}(N)=N\left\langle L_{F U V}\right\rangle_{*}
$$

Furthermore, the distribution of possible values for $L_{F U V}(N)$ must approach a gaussian form as $N \rightarrow \infty$ because of the central limit theorem (e.g., Richtmyer 1978), although convergence is slow. This gaussian form is (as usual) independent of the form of the initial distributions, i.e., it is 
independent of the stellar IMF and the mass-luminosity relation. The width of the distribution also converges to a known value and is given by

$$
\langle\sigma\rangle_{\text {fuv }}^{2}=\frac{1}{N} \sum_{j=1}^{N} \sigma_{j}^{2} \Rightarrow\langle\sigma\rangle_{\text {fuv }}=\sqrt{N} \sigma_{0},
$$

where $\sigma_{0}$ is the width of the individual distribution, i.e.,

$$
\sigma_{0}^{2} \equiv\left\langle L_{F U V}^{2}\right\rangle-\left\langle L_{F U V}\right\rangle_{*}^{2}
$$

For our usual choice of stellar properties, the dimensionless width $\widetilde{\sigma}_{0}=\sigma_{0} /\left\langle L_{F U V}\right\rangle_{*} \approx 26.4$, i.e., the variation in the possible values for the FUV luminosity is much greater than the expectation value. The effective "signal to noise ratio" $S / N$ for variations in FUV luminosity is thus given by

$$
S / N \equiv \frac{\langle\sigma\rangle_{\mathrm{fuv}}}{L_{F U V}(N)}=\frac{\sigma_{0}}{\sqrt{N}\left\langle L_{F U V}\right\rangle_{*}} \approx \frac{26.4}{\sqrt{N}}
$$

This definition thus defines a critical value of cluster members $N_{C}$, i.e., the number required for the variations in FUV luminosity to become sufficiently well-defined so that the variations are smaller than the expectation value. This critical value of $N_{C} \equiv \widetilde{\sigma}^{2} \sim 700$. For $N<N_{C}$, the FUV radiation experienced by a solar system living in the cluster is essentially determined by the largest star in the aggregeate; this largest member is, in turn, drawn from the probability distribution implied by the stellar IMF. For clusters with $N>N_{C}$, it makes sense to consider expectation values for the FUV radiation provided by the aggregate. However, the boundary is not sharp. Even for $N>N_{C}$, the total FUV luminosity $L_{F U V}(N)$ will be subject to substantial fluctuations from system to system.

We have performed a set of numerical sampling experiments to show that the mean and variance of the distribution agree with the analytic predictions derived above. The results are in good agreement. The mean and variance are shown as a function of cluster size $N$ in Figure 6 . These numerical experiments show that the convergence to a purely gaussian distribution is rather slow. The central value and variance of the actual distribution approach the values predicted by the central limit theorem much faster than the distribution itself approaches a normal form. As a result, the median of the distribution can be significantly different from the mean or expectation value, especially for cluster with small stellar membership $N$. Figure 6 also shows the median expected FUV flux as a function of cluster size $N$. The median is only about $8 \%$ of the mean for small clusters with $N \approx 100$ and approaches $80 \%$ of the mean for larger systems $(N=1000-2000)$. We note that the median value provides a better description of the "typical" FUV luminosity produced by a cluster of size $N$. However, the distribution of possibilities is wide and caution must be taken in characterizing the probability distribution by only one number.

Figure 7 shows the probability distributions for FUV luminosity for three choices of stellar size, namely $N=100,300$, and 1000 . The plot shows the cumulative probability distributions for the normalized FUV luminosity of the clusters, i.e., the total FUV luminosity divided by the number of cluster members $N$. In this representation, the distribution with $N=100$ (dashed curve) is 


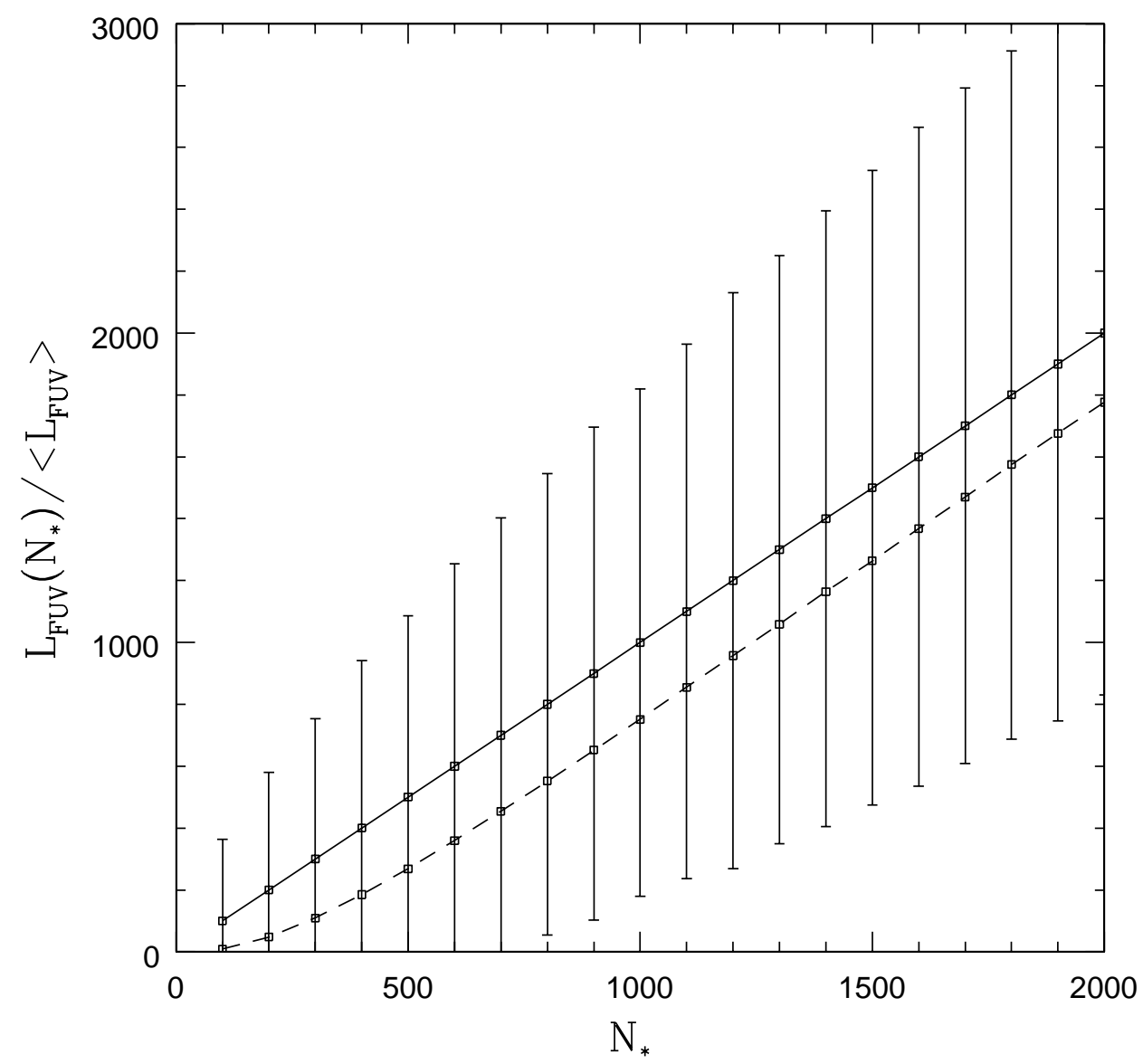

Fig. 6.- Mean, variance, and median of the distribution of total FUV luminosity for clusters of size $N$ as a function of $N$. The open square symbols connected by the solid line depict the mean FUV luminosity averaged over many realizations of a cluster of size $N$. The error bar symbols represent the variance about the mean. As shown in the text, the mean $\left\langle L_{F U V}\right\rangle \propto N$ and the variance $\sigma \propto N^{1 / 2}$. The median is shown by the dashed curve and is much smaller than the mean for small clusters, and slowly converges to the mean as $N \rightarrow \infty$. 


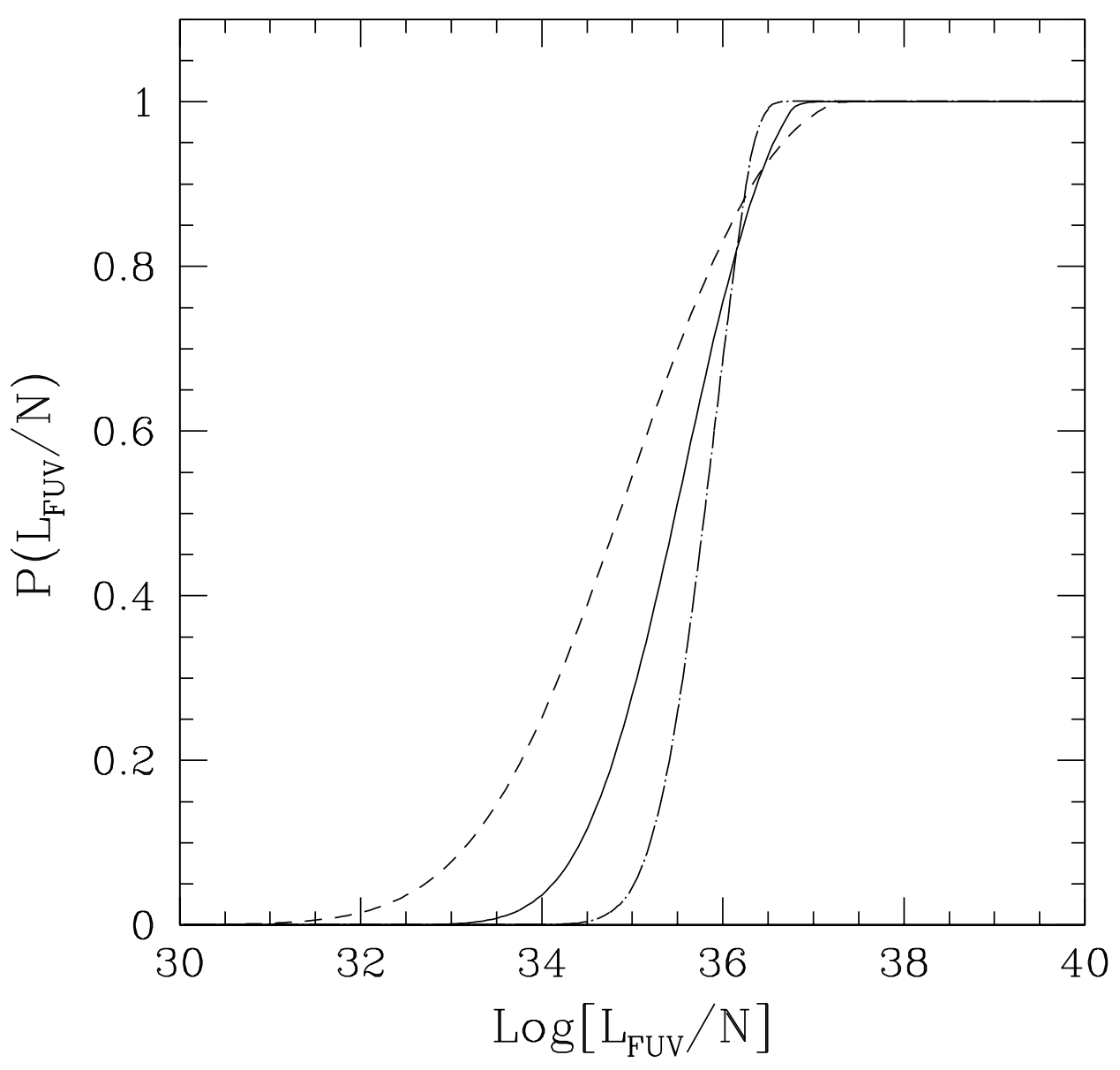

Fig. 7.- Cumulative probability distribution for the number of clusters that produce a given normalized FUV luminosity, where the normalized FUV luminosity is the total FUV luminosity of the cluster divided by the number $N$ of cluster members. The three curves shown correspond to clusters of size $N=100$ (dashed curve), $N=300$ (solid curve), and $N=1000$ (dot-dashed curve). 
the widest, and the distributions grow narrower with increasing $N$. In the limiting case $N \rightarrow \infty$, the distribution becomes a step function at the mean value $\left\langle L_{F U V}\right\rangle_{*}\left(\right.$ where $\left.\log _{10}\left\langle L_{F U V}\right\rangle_{*} \approx 35.9\right)$. For the three cluster sizes shown here, the normalized probability distributions converge near $P \approx$ 0.85 and $\log \left[L_{F U V} / N\right] \approx 36.2$. This convergence defines a benchmark for the high end of the distribution, namely, $15 \%$ of the clusters are expected to have FUV luminosity greater than the limit $L_{F U V} \geq N\left(1.6 \times 10^{36}\right) \mathrm{erg} / \mathrm{s} \approx 2 N\left\langle L_{F U V}\right\rangle_{*}$,

The distributions described above apply to clusters of a given size $N$ and show how the results depend on $N$. However, since stars are born in groups/clusters with a distribution of sizes $N$ (Figure 1, Lada \& Lada 2003, Porras et al. 2003), we also need to determine the overall distribution of FUV luminosities that affects the entire ensemble of forming solar systems. Toward that end, we assume that the distribution of stellar groups/clusters is the same as that of the Lada \& Lada (2003) sample (which is equivalent to that of Porras et al. 2003). We then sample the distribution of cluster systems to find a system size $N$, and for each such system we sample the IMF $N$ times to find the FUV luminosity. Using 10,000 realizations of the cluster sample (corresponding to a total of 127 million stars), we find the cumulative probability distribution for the expected FUV luminosity. The result is shown in Figure 8. This calculation shows that the median FUV

luminosity experienced by a forming solar system is $10^{38} \mathrm{erg} / \mathrm{s}$. This benchmark cluster luminosity is 122 times the mean FUV luminosity per star given by equation (12). If every star had the mean FUV luminosity, this result would imply a typical cluster size $N \approx 122$; since the typical (median) star has FUV luminosity less than the mean, the implied typical cluster size is somewhat larger (consistent with the distribution of Fig. 1). Notice also that individual stellar orbits within the cluster lead to different radiation exposure - this issue is discussed below.

\section{2. $\quad$ Effects of FUV Radiation}

The physical quantity that affects forming solar systems is the FUV flux, which depends on both the FUV luminosity (§3.1) and the radial position of the solar system within its birth aggregate $(\S 2)$. For relatively "small" clusters of interest in this paper, we can assume that the FUV luminosity originates from the few largest stars, which are generally observed to live near the cluster center (e.g., Testi, Palla, \& Natta 1999). Here we make the approximation that the FUV luminosity can be modeled as a point source at the origin. Any given solar system in orbit within a given cluster will thus experience a time dependent flux $\mathcal{F}_{F U V}=L_{F U V} / 4 \pi r^{2}$, where the radial position $r$ is specified by the orbit.

Each cluster provides a distribution of FUV fluxes to its cluster members, and each ensemble of clusters with a given size $N$ provides a wider distribution of FUV fluxes to the ensemble of members. Since clusters come in a distribution of sizes $N$, the collection of all forming solar systems is thus exposed to a wide distribution of FUV fluxes. Figure 9 shows an estimate for the probability distribution of FUV flux experienced by the entire ensemble of cluster stars as a function of flux. In this context, we express FUV flux in units of $G_{0}$, where $G_{0}=1$ corresponds to a benchmark 


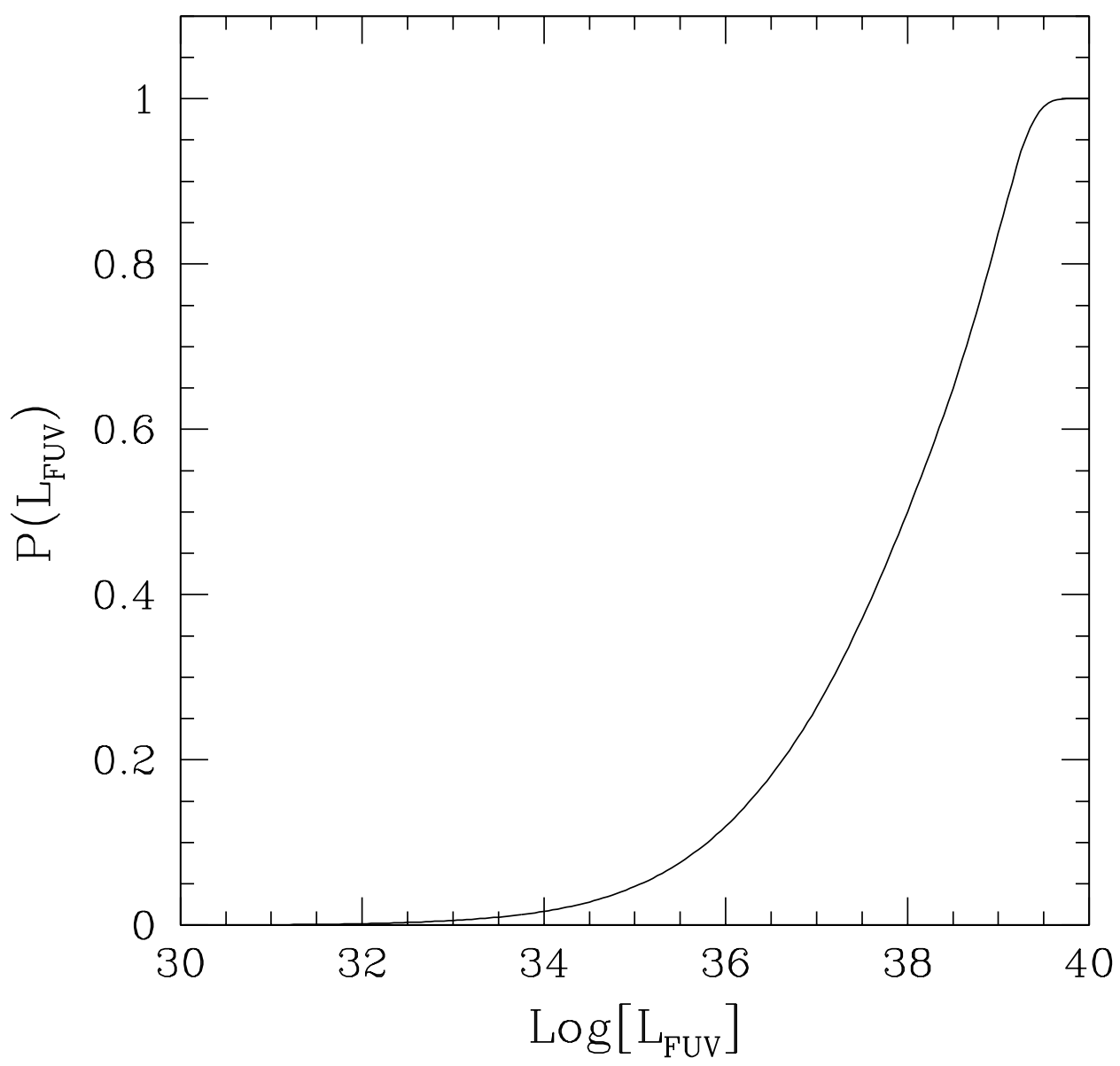

Fig. 8.- Cumulative probability distribution for the number of stars that live in a cluster with total FUV luminosity $L_{F U V}$ as a function of $L_{F U V}$. This distribution is calculated under the assumption that the cluster size distribution (the number of stars $N$ ) follows the data set of Lada \& Lada (2003). The size distribution of the data set is sampled 10,000 times to produce the probability distribution shown here. The median FUV luminosity occurs at $L_{F U V}=10^{38} \mathrm{erg} / \mathrm{s}$. Notice that the distribution has a long tail at low FUV luminosities. 
value of $1.6 \times 10^{-3} \mathrm{erg} \mathrm{s}^{-1} \mathrm{~cm}^{-2}$ at FUV wavelengths (close to the value of the interstellar radiation field). This ensemble distribution for the FUV flux was calculated assuming that the number of stars living in groups/clusters of size $N$ follows the distribution shown in Figure 1 (Lada \& Lada 2003; Porras et al. 2004), the radial size $R_{c *}$ of clusters follows the scaling relation shown in Figure 2 , the distribution of FUV luminosity follows that calculated in $\S 3.1$, and the density distribution within the cluster has the form $\rho_{*} \propto r^{-1}$ for $0 \leq r \leq R_{c *}$. The dashed curve in Figure 9 shows a gaussian distribution with the same peak location and the same FWHM; the true distribution has a substantial tail at low values and a much smaller tail at high flux values.

In Figure 9, the vertical lines at $G_{0}=300,3000$, and 30,000 are values for which the photoevaporation of circumstellar disks due to FUV radiation has been calculated in detail (Adams et al. 2004). In that study, the value $G_{0}=3000$ was chosen as a benchmark flux value (which corresponds to a cumulative probability of $\sim 0.74$ for the flux distribution found here). This FUV flux will drive the evaporation of a circumstellar disk around a $1.0 M_{\odot}$ star down to a radius of $r_{d} \approx 36 \mathrm{AU}$ in a time of $10 \mathrm{Myr}$. As a result, the region of the disk where giant planets form (5$30 \mathrm{AU})$ is relatively safe for solar type stars. For smaller stars, however, flux levels of $G_{0}=3000$ can be significant. A disk orbiting a $0.5 M_{\odot}\left(0.25 M_{\odot}\right)$ star can be evaporated down to $r_{d} \approx 18 \mathrm{AU}$ (9 AU) within 10 Myr. As a result, giant planet formation may be compromised around smaller stars (see also Laughlin et al. 2004). The results of the detailed photoevaporation models (Adams et al. 2004) can be summarized by a rough scaling law: An FUV flux of $G_{0}=3000$ truncates a circumstellar disk down to a radius $r_{d} \approx 36 \mathrm{AU}\left(M_{*} / M_{\odot}\right)$, over a time of $10 \mathrm{Myr}$.

The flux experienced by a "typical" star within a cluster can be characterized in a variety of ways. As shown by Figure 9, however, the distribution of flux values is extremely wide and cannot be fully described by a single number. If we consider the composite distribution of Figure 9 as representative, then the median FUV flux experienced by a forming solar system is $G_{0} \approx 900$, the peak of the distribution is at $G_{0} \approx 1800$, and the mean is at $G_{0} \approx 16,500$ (notice how the mean is much larger than the median).

We can also estimate the typical flux provided by a cluster of a given size $N$. This determination requires an integration over both the distribution of radial positions and the distribution of possible FUV luminosities. We can use either the mean or medians of these distributions as the "typical" values, but they lead to markedly different results, as shown in Table 5 below. For a given FUV luminosity, the mean radiation flux exposure is given by

$$
\left\langle\mathcal{F}_{F U V}\right\rangle=\frac{L_{F U V}}{4 \pi}\left\langle\frac{1}{r^{2}}\right\rangle
$$

where the average is taken over all stars and over all times. To evaluate $\left\langle r^{-2}\right\rangle$, we use the radial distributions $N(r)$ calculated from our numerical simulations (analogous to the mass profiles $M(r)$ given by eq. [3] and Table 2). We can write this expectation value in terms of an effective radius $r_{\text {eff }}$ defined by

$$
r_{e f f} \equiv\left\langle\frac{1}{r^{2}}\right\rangle^{-1 / 2}
$$




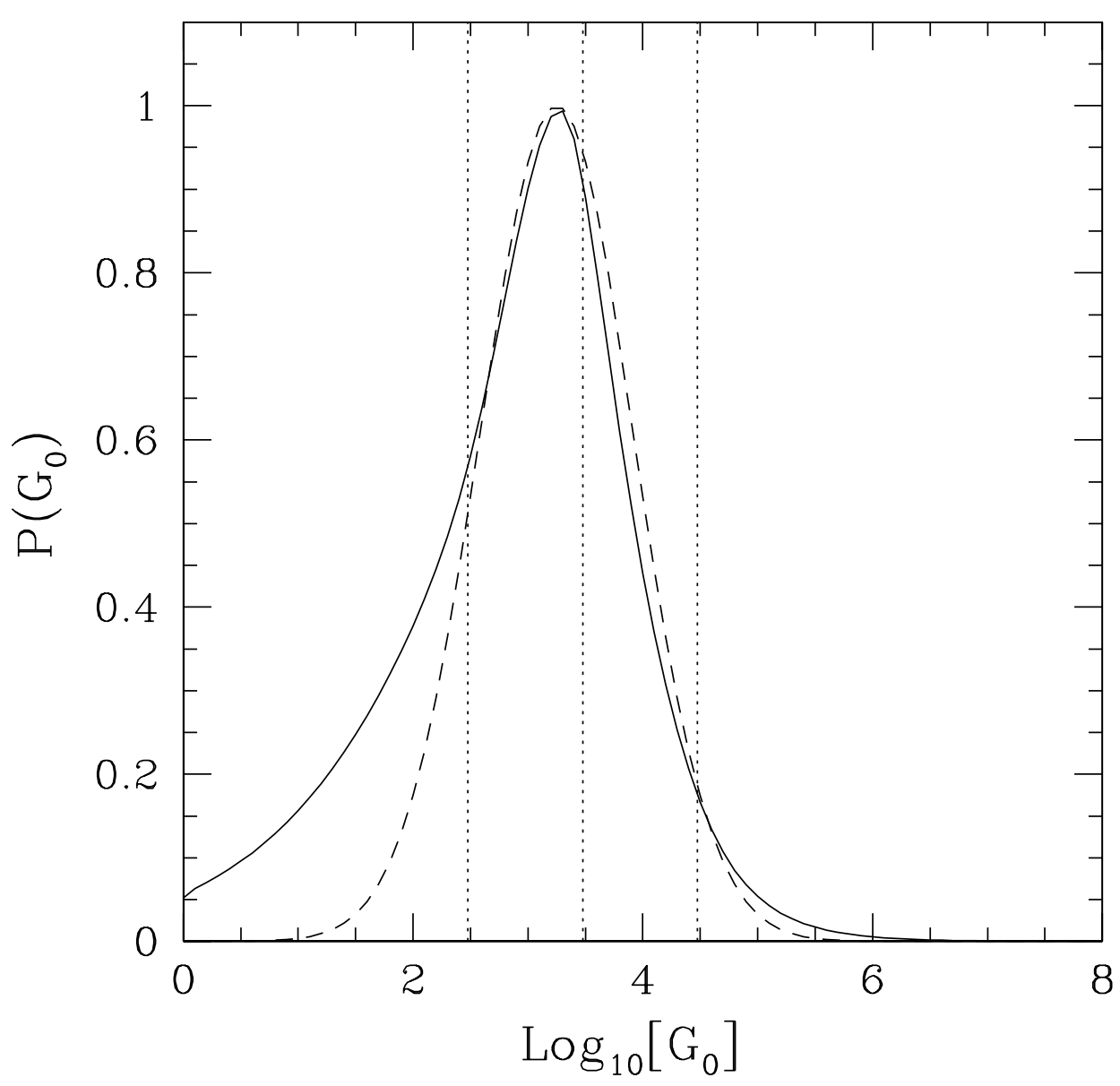

Fig. 9.- Probability distribution for FUV flux experienced by the ensemble of cluster stars as a function of the FUV flux, expressed here in units of $G_{0}$ (where $G_{0}=1$ correspondes to a flux of $1.6 \times 10^{-3} \mathrm{erg} \mathrm{s}^{-1} \mathrm{~cm}^{-2}$, which is close to the value of the interstellar radiation field). The ensemble distribution was calculated assuming that the number of stars living in groups/clusters of size $N$ follows the distribution of Figure 1, the radial size $R_{c *}$ of clusters follows the distribution shown in Figure 2, the distribution of FUV luminosity follows that calculated in $\S 3.1$, and the density distribution within the cluster has the simple form $\rho_{*} \propto r^{-1}$ for $0 \leq r \leq R_{c *}$. The dashed curve shows a gaussian distribution with the same peak location (at $\log _{10} G_{0}=3.25$ ) and the FWHM (1.575) The calculated distribution has a significant tail at low flux values. The vertical lines at $G_{0}=300,3000$, and 30000 are benchmark values for which the effects of photoevaporation on circumstellar disks has been calculated in detail (Adams et al. 2004). 
The effective radii for our six sets of simulations are given in Table 5. Using the mean values of the FUV luminosity in conjunction with the quantity $\left\langle r^{-2}\right\rangle$, we find a "mean" flux value, as listed in the table. Although this value provides one characterization of the FUV flux, the distributions are extremely wide, more than an order of magnitude wider than the mean value. As a result, most of the exposure occurs for those (relatively few) stars that wander close to the cluster center. The median thus provides a better measure of the typical FUV flux. The median values for the radial position, coupled with the median value of the FUV luminosity, provide an estimate for the "median" flux. The resulting values are also listed in Table 5. Notice that the flux levels calculated from the median values of radial position and FUV luminosity are much smaller than those calculated from the means (by factors of $17-190$ ). The mean and median values of the FUV flux, calculated from the composite distribution of Figure 9, are included as the final line in Table 5. Notice that the median flux of the composite $\left(G_{0}=900\right)$ is smaller than the median values for $N=1000$, larger than the values for $N=100$, and roughly comparable to the values for $N=300$. This ordering is consistent with finding that the median (weighted) value of cluster sizes occurs at $N \approx 300$ (from Figure 1), i.e., half of the stars in the sample are found in groups/clusters with $N<300$ and half are found in systems with $N>300$.

Table 5: Expected FUV Flux Values

\begin{tabular}{lcccc}
\hline \hline System & $\begin{array}{c}r_{\text {eff }} \\
(\mathrm{pc})\end{array}$ & $\begin{array}{c}G_{0} \\
\text {-mean- }\end{array}$ & $\begin{array}{c}r_{\text {med }} \\
(\mathrm{pc})\end{array}$ & $\begin{array}{c}G_{0} \\
\text {-median- }\end{array}$ \\
\hline 100 Cold & 0.080 & 66500 & 0.323 & 359 \\
100 Virial & 0.112 & 34300 & 0.387 & 250 \\
300 Cold & 0.126 & 81000 & 0.549 & 1550 \\
300 Virial & 0.181 & 39000 & 0.687 & 992 \\
1000 Cold & 0.197 & 109600 & 0.944 & 3600 \\
1000 Virial & 0.348 & 35200 & 1.25 & 2060 \\
\hline Composite & - & 16500 & - & 900 \\
\hline \hline
\end{tabular}

The distributions considered thus far describe the FUV flux experienced by forming solar systems in a statistical sense. A related question is to find the radiation experienced by a given solar system over the course of its orbit. Here we would like an analytic description of the orbits in order to see how the results depend on the relevant physical quantities. Toward this end, the mean flux intercepted by a solar system can be written in the form

$$
\left\langle\mathcal{F}_{F U V}\right\rangle=\frac{1}{\tau_{1 / 2}} \int \frac{L_{F U V}}{4 \pi r^{2}} d t
$$

where $\tau_{1 / 2}$ is the time of a half orbit (e.g., from the inner turning point to the outer turning point) and the integral is taken over that same time interval. Here we can model the orbits by assuming that the total cluster potential has the form of the Hernquist profile; orbits in this general class 
of extended mass distributions have a similar form (for further detail, see Adams \& Bloch 2005, hereafter AB05). The mean flux can then be written in the form

$$
\left\langle\mathcal{F}_{F U V}\right\rangle=\frac{\tau_{0}}{\tau_{1 / 2}} \frac{L_{F U V}}{4 \pi r_{s}^{2}} \int_{\xi_{1}}^{\xi_{2}} \frac{d t}{\xi^{2}}=\frac{\tau_{0}}{\tau_{1 / 2}} \frac{L_{F U V}}{4 \pi r_{s}^{2}}(\Delta \theta),
$$

where $\tau_{0} \equiv r_{s}\left(2 \Psi_{0}\right)^{-1 / 2}, \xi=r / r_{s}, r_{s}$ is the scale length of the Hernquist profile, $\xi_{j}$ are the turning points, and where $\Delta \theta \leq \pi$ is the angle subtended by the half orbit. The total depth of the gravitational potential well $\Psi_{0}$ is given by $\Psi_{0} \equiv G M_{T} / r_{s}$, where the mass scale $M_{T}=4 M_{1}$, where $M_{1}$ is the mass of the cluster (including both stars and gas) contained within the boundary $R_{c *}$. The orbit time and turning angle are known functions of the dimensionless energy $\epsilon \equiv|E| / \Psi_{0}$ and angular momentum variable $q \equiv j^{2} / 2 \Psi_{0} r_{s}^{2}$ (AB05). To within an accuracy of a few percent, one can express the dimensionless orbit time and the turning angle by the functions

$$
\frac{\tau_{1 / 2}}{\tau_{0}}=\epsilon^{-3 / 2}\left(\cos ^{-1} \sqrt{\epsilon}+\sqrt{\epsilon} \sqrt{1-\epsilon}\right),
$$

and

$$
\frac{\Delta \theta}{\pi}=\frac{1}{2}+\left[(1+8 \epsilon)^{-1 / 4}-\frac{1}{2}\right]\left[1+\frac{\ln \left(q / q_{\max }\right)}{6 \ln 10}\right]^{3.6},
$$

where $q_{\max }$ represents the maximum angular momentum for a given energy (that of a circular orbit) and the fitting function is restricted to the range $10^{-6} \leq q / q_{\max } \leq 1$. The maximum value of the angular momentum variable is also a known function of dimensionless energy,

$$
q_{\max }=\frac{1}{8 \epsilon} \frac{(1+\sqrt{1+8 \epsilon}-4 \epsilon)^{3}}{(1+\sqrt{1+8 \epsilon})^{2}} .
$$

Equations (20) - (23) thus specify the radiation exposure for a solar system on any orbit with given dimensionless energy $\epsilon$ and angular momentum $q$. The angular momentum dependence is relatively weak, much weaker than the dependence on energy. Since $\epsilon>3 / 8$ for orbits confined to the inner part of the Hernquist profile $(\xi<1)$, the turning angle is confined to the narrow range $(\Delta \theta) / \pi=1 / 2-\sqrt{2} / 2$. We can simplify the flux expression to take the form

$$
\left\langle\mathcal{F}_{F U V}\right\rangle=\frac{L_{F U V}}{8 r_{s}^{2}} \frac{A \epsilon^{3 / 2}}{\cos ^{-1} \sqrt{\epsilon}+\sqrt{\epsilon} \sqrt{1-\epsilon}},
$$

where the parameter $A$ is slowly varying and encapsulates the angular momentum dependence (with $1 \leq A \leq \sqrt{2}$ ). The leading coefficient sets the magnitude of the flux. The numerator can be written as $L_{F U V}=f_{N} N\left\langle L_{F U V}\right\rangle_{*}$, where $f_{N}$ is the fraction of the mean FUV luminosity that the cluster produces. For example, the median flux corresponds to $f_{N}=0.088$ for $N=100$ and $f_{N}=$ 0.75 for $N=1000$ (see Fig. 6), whereas the mean flux corresponds to $f_{N}=1$ by definition. In the denominator, the scale length $r_{s} \sim R_{c *}$, so that $r_{s}^{2} \sim(1 \mathrm{pc})^{2}(N / 300)$. The leading coefficient can thus be evaluated and written in terms of the interstellar FUV radiation field, i.e., $G \approx 2000 f_{N}$. The remaining dimensionless function in equation (20) is of order unity and accounts for the orbital shape. Deep orbits (close to the central part of the potential well) with $\epsilon \gtrsim 0.93$ have values of the 
dimensionless function greater than unity, whereas lower energy orbits have values less than unity. In any case, we find $G \sim 1000$, in agreement with the median values calculated above (Table 5).

The dynamics of the cluster determine the distributions of energy and angular momentum parameters $(\epsilon, q)$, and these distributions can be used in conjunction with equation (24) to calculate the distribution of fluxes experienced by young solar systems. For example, for an isotropic velocity distribution and a density profile $\rho \sim 1 / r$, the differential energy distribution (see BT87) has the form $h(\epsilon)=d m / d \epsilon \propto(1-\epsilon)$. Notice, however, that the flux distribution calculated from the distribution of energy $\epsilon$ (and $q$ ) is narrower than that calculated from the distribution of positions (that shown in Fig. 9). The fluxes in the former case are already averaged over the orbits, whereas those in the latter case are not and therefore explore a greater range of values.

\section{Scattering Interactions and the Disruption of Planetary Systems}

The ultimate goal of this section is to calculate the probability that a solar system will be disrupted as a result of being born within a group/cluster. In this case, disruption occurs through scattering interactions with other cluster members, and should thus depend on the size $N$ of the birth aggregate. In general, the rate of disruption for a solar system can be written in the form

$$
\Gamma=n \sigma v,
$$

where $\sigma$ is the disruption cross section, $n$ is the mean density of other systems, and $v$ is the relative velocity (typically, $v \sim 1 \mathrm{~km} / \mathrm{s}$ ). In this case, however, the results of $N$-body simulations provide the rate at which solar system experience close encounters within a closest approach distance $b$ as a function of $b$. As a result, we only need to determine how close such scattering encounters must be in order for disruption to take place. Here we make the approximation that the cluster dynamics (which determines the rate of close encounters) is independent of the scattering dynamics between a solar system and a passing star (which determines the cross sections).

Using our planet scattering code developed previously (Laughlin \& Adams 2000; Adams \& Laughlin 2001), we can calculate the cross sections for the disruption of solar systems with varying stellar masses (see also Heller 1993, 1995; de la Fuenta Marcos \& de la Fuente Marcos 1997, 1999). Since most stars passing nearby with the potential for disrupting a solar system are binaries, we want to find the effective cross section $\langle\sigma\rangle$ for a specified change in orbital parameters resulting from scattering encounters with binaries. We define this effective cross section $\langle\sigma\rangle$ through the relation

$$
\langle\sigma\rangle \equiv \int_{0}^{\infty} f_{D}(a)\left(4 \pi a^{2}\right) p(a) d a,
$$

where $a$ is the semimajor axis of the binary orbit and $p(a)$ specifies the probability of encountering a binary system with a given value of $a$. Notice that for a given value of $a$, this integal only includes those scattering interactions that fall within the predetermined area $4 \pi a^{2}$, where the numerical coefficient (4) is chosen to be large enough to include all relevant interactions and small enough to 
allow for finite computing resources (in principle, one should include all interactions, no matter how distant). In practice, we find that the area $4 \pi a^{2}$ provides a good compromise between accuracy and computational expediency. The function $f_{D}(a)$ specifies the fraction of the encounters that result in a particular outcome (e.g., a given change in the orbital parameters of the solar system), and the determination of $f_{D}$ is the main computational task. The distribution $p(a)$ is determined by the observed distribution of binary periods and by the normalization condition

$$
\int_{0}^{\infty} p(a) d a=1
$$

where we use observational results to specify the period distribution (Kroupa 1995a). The observed distribution is extremely broad, with roughly equal numbers of systems in each logarithmic interval in semimajor axis $a$ and with a broad overall peak falling near $P=10^{5}$ days. Although the distribution includes binaries with periods longer than $10^{7}$ days, this set of scattering experiments only includes binaries with $a<1000$ AU because wider binaries do not contribute appreciably to the cross sections.

The set of possible encounters that can occur between a given solar system and a field binary is described by 10 basic input parameters. These variables include the binary semimajor axis $a$, the stellar masses, $m_{* 1}$ and $m_{* 2}$, of the binary pair, the eccentricity $e_{\mathrm{b}}$ and the initial phase angle $\ell_{\mathrm{b}}$ of the binary orbit, the asymptotic incoming velocity $v_{\text {inf }}$ of the solar system with respect to the center of mass of the binary, the angles $\theta, \psi$, and $\phi$ which describe the impact direction and orientation, and finally the impact parameter $h$ of the collision. Additional (intrinsic) parameters are required to specify the angular momentum vector and initial orbital phases of the planets within the solar system. In this study, we consider a class of solar systems in which the central stellar mass varies, but the planetary orbits are always taken to be those of our solar system. In other words, each solar system has an analog of Jupiter, a planet with one Jupiter mass $m_{J}$ in an initial orbit of semimajor axis $a_{J}=5 \mathrm{AU}$; similarly, each solar system has an analog of Saturn, Uranus, and Neptune. The planetary orbits are assumed to be circular (initially) so that we can determine the change in orbital parameters from a known baseline. All of the planetary properties are kept fixed so we can isolate the effects of changing the mass of the central star.

In order to compute the fraction of disruptive encounters $f_{D}(a)$ and the corresponding cross sections, we perform a large number of scattering experiments using a Monte Carlo scheme to select the input parameters. This section reports on the results from more than $10^{5}$ such scattering experiments. Individual encounters are treated as $N$-body problems in which the equations of motion are integrated using a Bulirsch-Stoer scheme (Press et al. 1986). During each encounter, we require that overall energy conservation be maintained to an accuracy of at least one part in $10^{8}$. For most experiments, energy and angular momentum are conserved to one part in $10^{10}$. This high level of accuracy is needed because we are interested in the resulting planetary orbits, which carry only a small fraction of the total angular momentum and orbital energy of the system.

For each scattering experiment, the initial conditions are drawn from the appropriate parameter distributions. More specifically, the binary eccentricities are sampled from the observed distribution 
(Duquennoy \& Mayor 1991). The masses of the two binary components are drawn separately from a log-normal initial mass function (IMF) that is consistent with the observed distribution of stellar masses (for completeness, we note that the IMF has a power-law tail at high masses, although this departure will not affect the cross sections calculated here). For both the primary and the secondary, we enforce a lower mass limit of $0.075 M_{\odot}$ and hence our computed scattering results do not include brown dwarfs. This cutoff has a relatively small effect because our assumed IMF peaks in the stellar regime. The impact velocities at infinite separation, $v_{\text {inf }}$, are sampled from a Maxwellian distribution with dispersion $\sigma_{v}=1 \mathrm{~km} / \mathrm{s}$, which is a typical value for stellar clusters with the range of parameters considered here (BT87). The initial impact parameters $h$ are chosen randomly within a circle of radius $2 a$ centered on the binary center of mass (a circular target area of $4 \pi a^{2}$ as in eq. [26]).

Using the Monte Carlo technique outlined above, we have performed $N_{\exp } \approx 20,000$ scattering experiments for collisions between binary star systems and solar systems of each stellar mass (i.e., a total of $\sim 10^{5}$ simulations). We use logarithmically spaced mass values: $M_{*} / M_{\odot}=2,1,1 / 2,1 / 4$, and $1 / 8$. As described above, all solar systems are taken to have the architecture of our outer solar system. From the results of these 7-body scattering experiments, we compute the cross sections for orbital disruption of each outer planet (according to equation [26]). Note that the procedure described thus far implicitly asssumes that all passing stars are binary. Although the majority of solar type stars have binary companions, smaller stars (e.g., M stars) have a lower binary fraction. The true cross sections should thus be written as $\langle\sigma\rangle_{T}=F_{b}\langle\sigma\rangle_{\mathrm{b}}+\left(1-F_{b}\right)\langle\sigma\rangle_{\mathrm{s}}$, where $F_{b}$ is the binary fraction and $\langle\sigma\rangle_{\mathrm{s}}$ is the cross section of interactions between single stars and solar systems (and is not calculated here). However, $\langle\sigma\rangle_{\mathrm{s}} \ll\langle\sigma\rangle_{\mathrm{b}}$ (Adams \& Laughlin 2001) so that to a good working approximation one can use $\langle\sigma\rangle_{T} \approx F_{b}\langle\sigma\rangle_{\mathrm{b}}$.

The cross sections for the planets to increase their orbital eccentricities are shown in Tables 6 - 10 for varying stellar mass (see also Fig. 10). For each given value of eccentricity $e$, the table entries give the cross sections [in units of $(\mathrm{AU})^{2}$ ] for the eccentricity to increase to any value greater than the given $e$; these cross sections include events leading to either ejection of the planet or capture by another star. The listings for $e=1$ thus give the total cross sections for planetary escape and capture (taken together). The last two lines of the Tables present the cross sections for planetary escapes and captures separately. For each cross section listed, the Tables also provide the one standard deviation error estimate [also in $(\mathrm{AU})^{2}$ ] for the Monte Carlo integral; this quantity provides a rough indication of the errors due to the statistical sampling process (Press et al. 1986). Figure 10 shows the cross sections plotted as a function of the eccentricity increase for each of the four planets and for the four largest stellar mass values. 

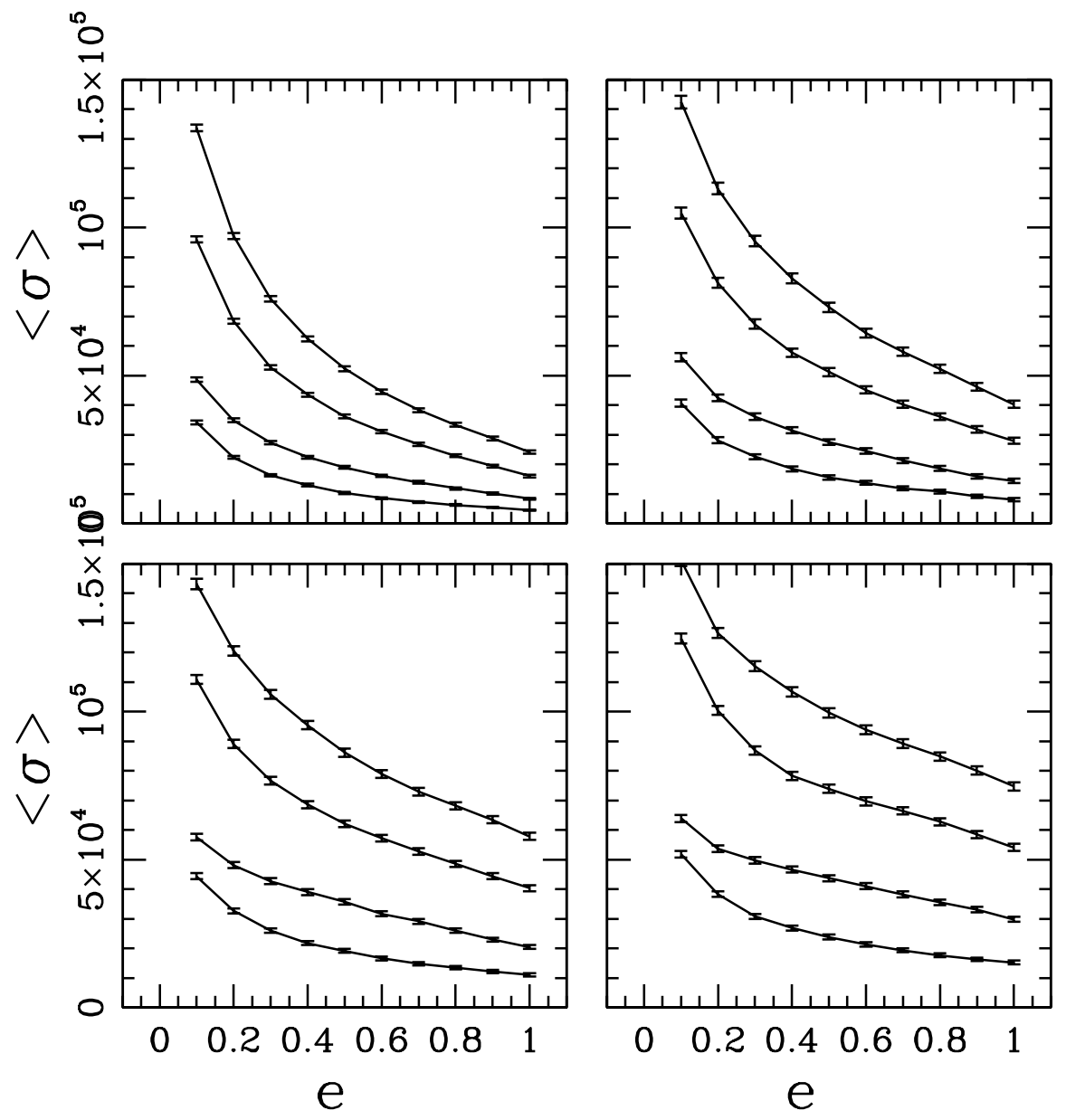

Fig. 10.- Scattering cross sections for solar systems to increase the eccentricity $e$ of planetary orbits, plotted here as a function of eccenctricity. All cross sections are given in units of (AU) ${ }^{2}$. The four panels shown here correspond to the four largest stellar mass values of our computational survey, i.e., $M_{*}=2.0 M_{\odot}$ (upper left) $1.0 M_{\odot}$ (upper right), $0.5 M_{\odot}$ (lower left), and $0.25 M_{\odot}$ (lower right). In each panel, the four curves shown correspond to four giant planets orbiting the central star, where the planets have the same masses and starting semimajor axes as the giant planets in our solar system. The top curve in each panel corresponds to an analog of Neptune and the bottom curve corresponds to an analog of Jupiter. The cross section for increasing the eccentricity beyond unity (right end points of the curves) corresponds to ejection of the planet. The error bars correspond to the uncertainties incurred due to the (incomplete) Monte Carlo sampling of the parameter space. 
Table 6: Scattering Cross Sections for $M_{*}=2.0 M_{\odot}$ Stars

\begin{tabular}{lcccc}
\hline \hline$e /$ outcome & $\langle\sigma\rangle$ (Jupiter) & $\langle\sigma\rangle$ (Saturn) & $\langle\sigma\rangle$ (Uranus) & $\langle\sigma\rangle$ (Neptune) \\
\hline 0.10 & $34200 \pm 632$ & $48600 \pm 738$ & $96000 \pm 1030$ & $133700 \pm 1180$ \\
0.20 & $22300 \pm 513$ & $34900 \pm 631$ & $68400 \pm 881$ & $97200 \pm 1040$ \\
0.30 & $16300 \pm 436$ & $27300 \pm 563$ & $52800 \pm 782$ & $75900 \pm 926$ \\
0.40 & $13000 \pm 389$ & $22400 \pm 511$ & $43500 \pm 714$ & $62400 \pm 846$ \\
0.50 & $10300 \pm 343$ & $18900 \pm 471$ & $36100 \pm 653$ & $52300 \pm 780$ \\
0.60 & $8560 \pm 314$ & $16100 \pm 439$ & $31100 \pm 609$ & $44600 \pm 725$ \\
0.70 & $7300 \pm 291$ & $13900 \pm 410$ & $26800 \pm 569$ & $38300 \pm 677$ \\
0.80 & $6240 \pm 269$ & $11900 \pm 380$ & $22900 \pm 526$ & $33300 \pm 635$ \\
0.90 & $5470 \pm 253$ & $10100 \pm 351$ & $19400 \pm 486$ & $28700 \pm 595$ \\
1.00 & $4550 \pm 231$ & $8350 \pm 321$ & $16000 \pm 446$ & $24200 \pm 555$ \\
escape & $4010 \pm 216$ & $7590 \pm 306$ & $13900 \pm 413$ & $20600 \pm 504$ \\
capture & $539 \pm 83$ & $764 \pm 99$ & $2100 \pm 168$ & $3640 \pm 232$ \\
\hline \hline
\end{tabular}

Table 7: Scattering Cross Sections for $M_{*}=1.0 M_{\odot}$ Stars

\begin{tabular}{lcccc}
\hline \hline$e /$ outcome & $\langle\sigma\rangle$ (Jupiter) & $\langle\sigma\rangle$ (Saturn) & $\langle\sigma\rangle$ (Uranus) & $\langle\sigma\rangle$ (Neptune) \\
\hline 0.10 & $40700 \pm 1190$ & $56300 \pm 1380$ & $104900 \pm 1860$ & $142400 \pm 2110$ \\
0.20 & $28100 \pm 996$ & $42500 \pm 1200$ & $81300 \pm 1650$ & $113200 \pm 1910$ \\
0.30 & $22600 \pm 895$ & $36100 \pm 1110$ & $67400 \pm 1510$ & $95400 \pm 1780$ \\
0.40 & $18500 \pm 801$ & $31500 \pm 1040$ & $57700 \pm 1400$ & $82900 \pm 1670$ \\
0.50 & $15500 \pm 731$ & $27500 \pm 974$ & $51200 \pm 1330$ & $73100 \pm 1580$ \\
0.60 & $13700 \pm 692$ & $24500 \pm 924$ & $45200 \pm 1250$ & $64300 \pm 1480$ \\
0.70 & $11900 \pm 640$ & $21300 \pm 865$ & $40300 \pm 1190$ & $58000 \pm 1420$ \\
0.80 & $10800 \pm 606$ & $18600 \pm 812$ & $36100 \pm 1130$ & $52200 \pm 1360$ \\
0.90 & $9270 \pm 564$ & $16000 \pm 754$ & $31800 \pm 1060$ & $46200 \pm 1280$ \\
1.00 & $8040 \pm 529$ & $14400 \pm 728$ & $28000 \pm 1010$ & $40300 \pm 1220$ \\
escape & $7060 \pm 488$ & $13100 \pm 688$ & $24900 \pm 952$ & $33900 \pm 1100$ \\
capture & $973 \pm 203$ & $1350 \pm 238$ & $3010 \pm 347$ & $6390 \pm 517$ \\
\hline \hline
\end{tabular}


Table 8: Scattering Cross Sections for $M_{*}=0.50 M_{\odot}$ Stars

\begin{tabular}{lcccc}
\hline \hline$e /$ outcome & $\langle\sigma\rangle$ (Jupiter) & $\langle\sigma\rangle$ (Saturn) & $\langle\sigma\rangle$ (Uranus) & $\langle\sigma\rangle$ (Neptune) \\
\hline 0.10 & $44400 \pm 1010$ & $57700 \pm 1140$ & $110900 \pm 1560$ & $143200 \pm 1730$ \\
0.20 & $32700 \pm 870$ & $48200 \pm 1050$ & $89100 \pm 1400$ & $120600 \pm 1610$ \\
0.30 & $26000 \pm 775$ & $42800 \pm 993$ & $76600 \pm 1310$ & $105900 \pm 1520$ \\
0.40 & $21800 \pm 708$ & $39100 \pm 952$ & $68600 \pm 1250$ & $95500 \pm 1460$ \\
0.50 & $19200 \pm 664$ & $35800 \pm 914$ & $62200 \pm 1190$ & $86200 \pm 1390$ \\
0.60 & $16600 \pm 618$ & $31700 \pm 860$ & $57300 \pm 1150$ & $78900 \pm 1340$ \\
0.70 & $14800 \pm 585$ & $29200 \pm 828$ & $52900 \pm 1110$ & $72900 \pm 1290$ \\
0.80 & $13500 \pm 557$ & $26000 \pm 782$ & $48600 \pm 1070$ & $68200 \pm 1260$ \\
0.90 & $12200 \pm 531$ & $23000 \pm 732$ & $44400 \pm 1020$ & $63500 \pm 1220$ \\
1.00 & $11100 \pm 510$ & $20500 \pm 693$ & $40400 \pm 982$ & $57900 \pm 1190$ \\
escape & $10400 \pm 496$ & $18900 \pm 665$ & $36200 \pm 922$ & $50600 \pm 1090$ \\
capture & $701 \pm 119$ & $1560 \pm 194$ & $4150 \pm 338$ & $7300 \pm 458$ \\
\hline \hline
\end{tabular}

Table 9: Scattering Cross Sections for $M_{*}=0.25 M_{\odot}$ Stars

\begin{tabular}{lcccc}
\hline \hline$e /$ outcome & $\langle\sigma\rangle$ (Jupiter) & $\langle\sigma\rangle$ (Saturn) & $\langle\sigma\rangle$ (Uranus) & $\langle\sigma\rangle$ (Neptune) \\
\hline 0.10 & $51900 \pm 1140$ & $63900 \pm 1250$ & $124800 \pm 1720$ & $151100 \pm 1850$ \\
0.20 & $38400 \pm 979$ & $53800 \pm 1150$ & $100500 \pm 1560$ & $126700 \pm 1710$ \\
0.30 & $30900 \pm 875$ & $49700 \pm 1110$ & $86900 \pm 1460$ & $115400 \pm 1650$ \\
0.40 & $26900 \pm 810$ & $46700 \pm 1080$ & $78300 \pm 1390$ & $106800 \pm 1600$ \\
0.50 & $23900 \pm 761$ & $43800 \pm 1050$ & $74000 \pm 1350$ & $99700 \pm 1550$ \\
0.60 & $21300 \pm 717$ & $41100 \pm 1020$ & $69700 \pm 1320$ & $93900 \pm 1510$ \\
0.70 & $19400 \pm 683$ & $38300 \pm 988$ & $66500 \pm 1290$ & $89300 \pm 1480$ \\
0.80 & $17700 \pm 655$ & $35600 \pm 959$ & $62800 \pm 1260$ & $84900 \pm 1450$ \\
0.90 & $16300 \pm 630$ & $33200 \pm 929$ & $58600 \pm 1220$ & $80200 \pm 1410$ \\
1.00 & $15300 \pm 614$ & $29900 \pm 880$ & $54100 \pm 1190$ & $74800 \pm 1400$ \\
escape & $14500 \pm 597$ & $27800 \pm 842$ & $49300 \pm 1120$ & $65300 \pm 1290$ \\
capture & $741 \pm 140$ & $2040 \pm 257$ & $4880 \pm 394$ & $9440 \pm 546$ \\
\hline \hline
\end{tabular}


Table 10: Scattering Cross Sections for $M_{*}=0.125 M_{\odot}$ Stars

\begin{tabular}{lcccc}
\hline \hline$e /$ outcome & $\langle\sigma\rangle$ (Jupiter) & $\langle\sigma\rangle$ (Saturn) & $\langle\sigma\rangle$ (Uranus) & $\langle\sigma\rangle$ (Neptune) \\
\hline 0.10 & $65100 \pm 1170$ & $77700 \pm 1260$ & $147500 \pm 1690$ & $208200 \pm 1940$ \\
0.20 & $50200 \pm 1030$ & $67800 \pm 1190$ & $111600 \pm 1480$ & $134700 \pm 1590$ \\
0.30 & $37600 \pm 888$ & $63800 \pm 1160$ & $99400 \pm 1410$ & $121400 \pm 1530$ \\
0.40 & $30800 \pm 796$ & $61500 \pm 1140$ & $92400 \pm 1370$ & $113600 \pm 1490$ \\
0.50 & $26700 \pm 734$ & $58900 \pm 1120$ & $87200 \pm 1340$ & $107200 \pm 1450$ \\
0.60 & $24000 \pm 694$ & $56000 \pm 1100$ & $83100 \pm 1310$ & $101800 \pm 1420$ \\
0.70 & $21800 \pm 664$ & $52700 \pm 1070$ & $79100 \pm 1280$ & $97800 \pm 1400$ \\
0.80 & $20000 \pm 637$ & $49300 \pm 1040$ & $75100 \pm 1250$ & $93800 \pm 1380$ \\
0.90 & $18600 \pm 612$ & $45200 \pm 1000$ & $72100 \pm 1230$ & $90100 \pm 1350$ \\
1.00 & $17200 \pm 588$ & $40100 \pm 948$ & $65900 \pm 1190$ & $84700 \pm 1340$ \\
escape & $16100 \pm 564$ & $38000 \pm 920$ & $60100 \pm 1130$ & $74500 \pm 1240$ \\
capture & $1060 \pm 167$ & $2160 \pm 231$ & $5750 \pm 386$ & $10200 \pm 524$ \\
\hline \hline
\end{tabular}

Table 11: Scattering Cross Sections for Angular Increase

\begin{tabular}{cccccc}
\hline \hline$\Delta i$ & $\langle\sigma\rangle$ & $\langle\sigma\rangle$ & $\langle\sigma\rangle$ & $\langle\sigma\rangle$ & $\langle\sigma\rangle$ \\
(degrees) & $0.125 M_{\odot}$ & $0.25 M_{\odot}$ & $0.5 M_{\odot}$ & $1.0 M_{\odot}$ & $2.0 M_{\odot}$ \\
\hline 10.0 & 108800 & 104400 & 97400 & 89600 & 75500 \\
20.0 & 91100 & 85600 & 78000 & 65800 & 50700 \\
30.0 & 83200 & 77300 & 68000 & 55100 & 40400 \\
40.0 & 77600 & 71900 & 61600 & 48800 & 34400 \\
50.0 & 72800 & 67600 & 55900 & 43700 & 30200 \\
60.0 & 68000 & 62500 & 51900 & 40100 & 26500 \\
70.0 & 63000 & 57400 & 47900 & 36500 & 24000 \\
80.0 & 58300 & 53000 & 43600 & 33700 & 21800 \\
90.0 & 54100 & 48600 & 40300 & 30700 & 19400 \\
\hline \hline
\end{tabular}

Another way for solar systems to be disrupted is by changing the planes of the planetary orbits. One can use the results of the Monte Carlo scattering calculations to compute the cross sections for the inclination angles of the planetary orbits to increase by varying amounts. These results are shown as a function of angle in Table 11 for the five stellar mass values used here. More specifically, we define the inclination angle increase $\Delta i$ to be the maximum angle between the orbital plane of the perturbed (post-encounter) planets and the original orbital plane. In Table 11, the Monte Carlo uncertainties are not listed, but the sampling statistics are good, and the effective errors are approximately 2 percent. In addition, we find that the increases in inclination angle are well correlated with the predicted increases in eccentricity, as shown in Figure 11. For the five stellar 
masses considered here, the linear correlation coefficient between the inclination angle increase and the (final) eccentricity of the Neptune-analog planet lies in the range $\mathcal{R}=0.70-0.75$.

The cross sections scale roughly with the inverse square root of the stellar mass. For example, the total ejection cross section is one of the more useful quantities considered here. We find that the mass dependence of the cross section for a given planet to be ejected can be written in the form

$$
\langle\sigma\rangle_{\mathrm{ej}}\left(M_{*} / M_{\odot}\right)^{1 / 2} \approx \mathcal{C}_{P} \approx \text { constant }
$$

where the constant $\mathcal{C}_{P}$ depends on which planet is being ejected. For Jupiter, Saturn, Uranus, and Neptune, respectively, we find $\mathcal{C}_{J}=7200 \pm 800 \mathrm{AU}^{2}, \mathcal{C}_{S}=14,000 \pm 1100 \mathrm{AU}^{2}, \mathcal{C}_{U}=25,900 \pm$ $2440 \mathrm{AU}^{2}$, and $\mathcal{C}_{N}=36,600 \pm 4070 \mathrm{AU}^{2}$. When we scale the cross sections by the mass of the central star, the scaling law is not perfect, but rather retains some variation that is quantified by the quoted "error bars" given here. Next we note that these cross sections almost scale linearly with the semimajor axes of the planet. If we perform such a scaling, the ejection cross section can be written in the form

$$
\langle\sigma\rangle_{\mathrm{ej}} \approx \mathcal{C}_{0}\left(a_{p} / \mathrm{AU}\right)\left(M_{*} / M_{\odot}\right)^{-1 / 2}
$$

where $\mathcal{C}_{0}=1350 \pm 160 \mathrm{AU}^{2}$ and where $a_{p}$ is the semimajor axis of the planetary orbit.

Now we can put the pieces together and apply these results to clusters. The output measures from the numerical simulations show that the rates of close encounters have the form $\Gamma=\Gamma_{0}\left(r / r_{0}\right)^{\gamma}$, where the parameters $\Gamma_{0}$ and $\gamma$ depend on the starting conditions in the cluster. The length scale $r_{0}=1000 \mathrm{AU}$ defines the units. The rate of ejection of planets is thus given by

$$
\Gamma_{\text {eject }}=\Gamma_{0}\left[\frac{\mathcal{C}_{0}\left(a_{p} / \mathrm{AU}\right)}{\pi r_{0}^{2}}\right]^{\gamma / 2}\left(\frac{M_{*}}{M_{\odot}}\right)^{-\gamma / 4} .
$$

This expression gives the ejection rate per star for a given $M_{*}$. To find the total ejection rate for the cluster, one must integrate over the IMF, normalized to the cluster size $N$, i.e., $\int d m(d N / d m) m^{-\gamma / 4}$ where $\int(d N / d m) d m=N$. For example, the rate of ejection of planetary analogs of Jupiter in a cluster of $N=300$ stars with a cold starting condition can be readily found: The numerical simulations provide $\Gamma_{0}=0.096$ (interactions per star per Myr) and $\gamma=1.7$ (see Table 3). The ejection rate of Jupiters is thus $\Gamma_{J} \approx 0.15$ ejections per cluster per Myr. Over the 10 Myr lifetime spanned by the simulations, only 1 or 2 Jupiter ejections are expected per cluster (these results are consistent with those obtained by Smith \& Bonnell 2001 and by de le Fuente Marcos \& de la Fuente Marcos 1997). The number of ejected planets is not only small, but it is much smaller than the number of ejections expected from internal (planet-planet) scattering events (Moorhead \& Adams 2005). Even for the larger cross section for the ejection of Neptunes, the number of expected events is only about 7 . For solar systems orbiting smaller stars, e.g., with mass $M_{*}=0.25$ $M_{\odot}$, the ejection cross sections and hence the expected number of ejected planets are larger by a factor of $\sim 2$. Of course, smaller stars may have trouble forming planets due to increased efficacy of disk evaporation (§3, Adams et al. 2004) and other difficulties (Laughlin et al. 2004). 


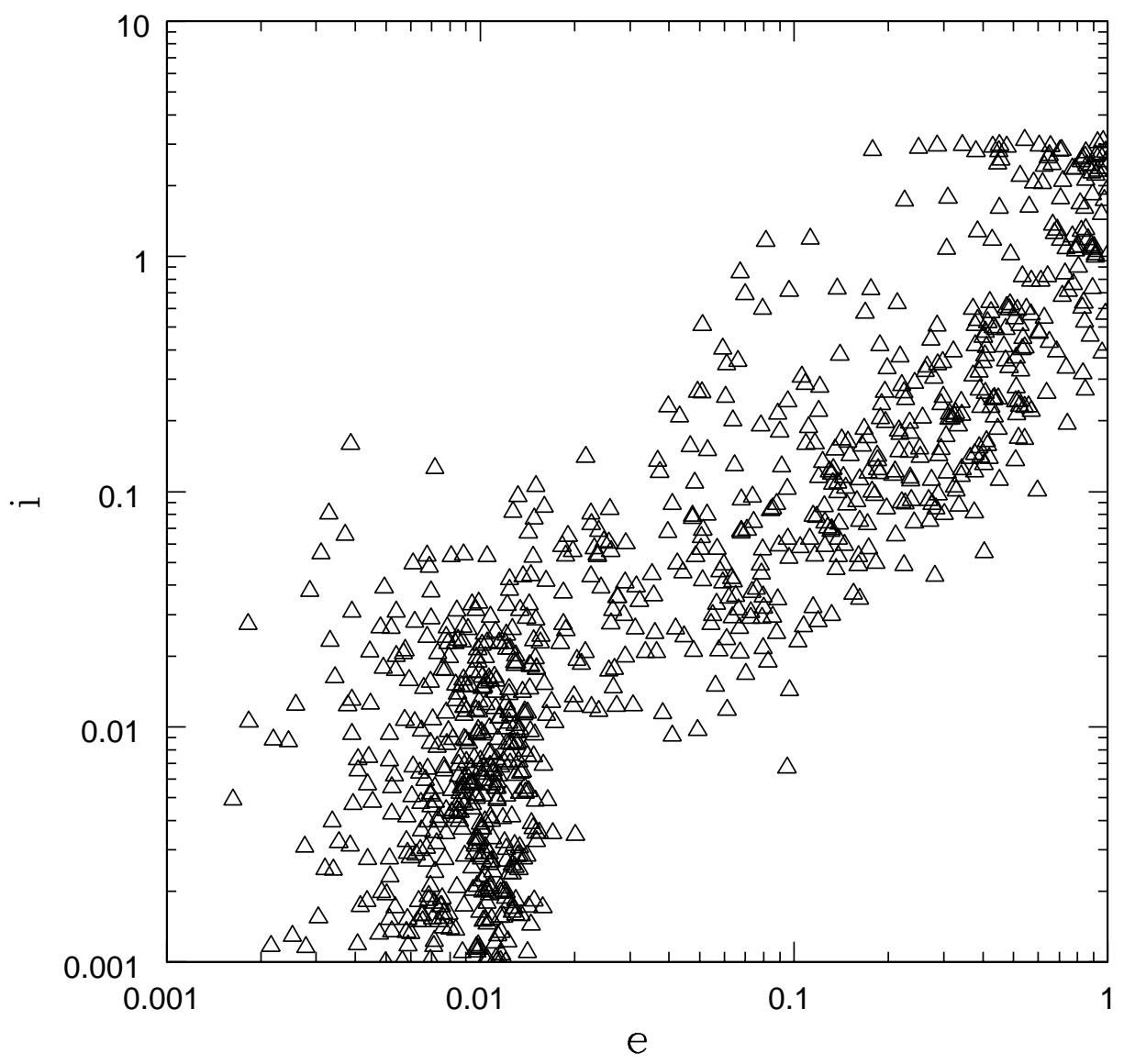

Fig. 11. - Scatter plot showing the correlation of changes in eccentricity with changes in the orbital inclination angle (in radians). Each symbol shows the result of one scattering simulation (not all cases are shown). The inclination angle is defined to be the maximum angle between the the perturbed orbital planes of the planets and the original orbital plane. The eccentricity considered here is the (final) eccentricity of the Neptune analog. This plot shows results from the scattering experiments using stellar mass $M_{*}=1.0 M_{\odot}$; the results are similar for all five stellar masses considered herein. 


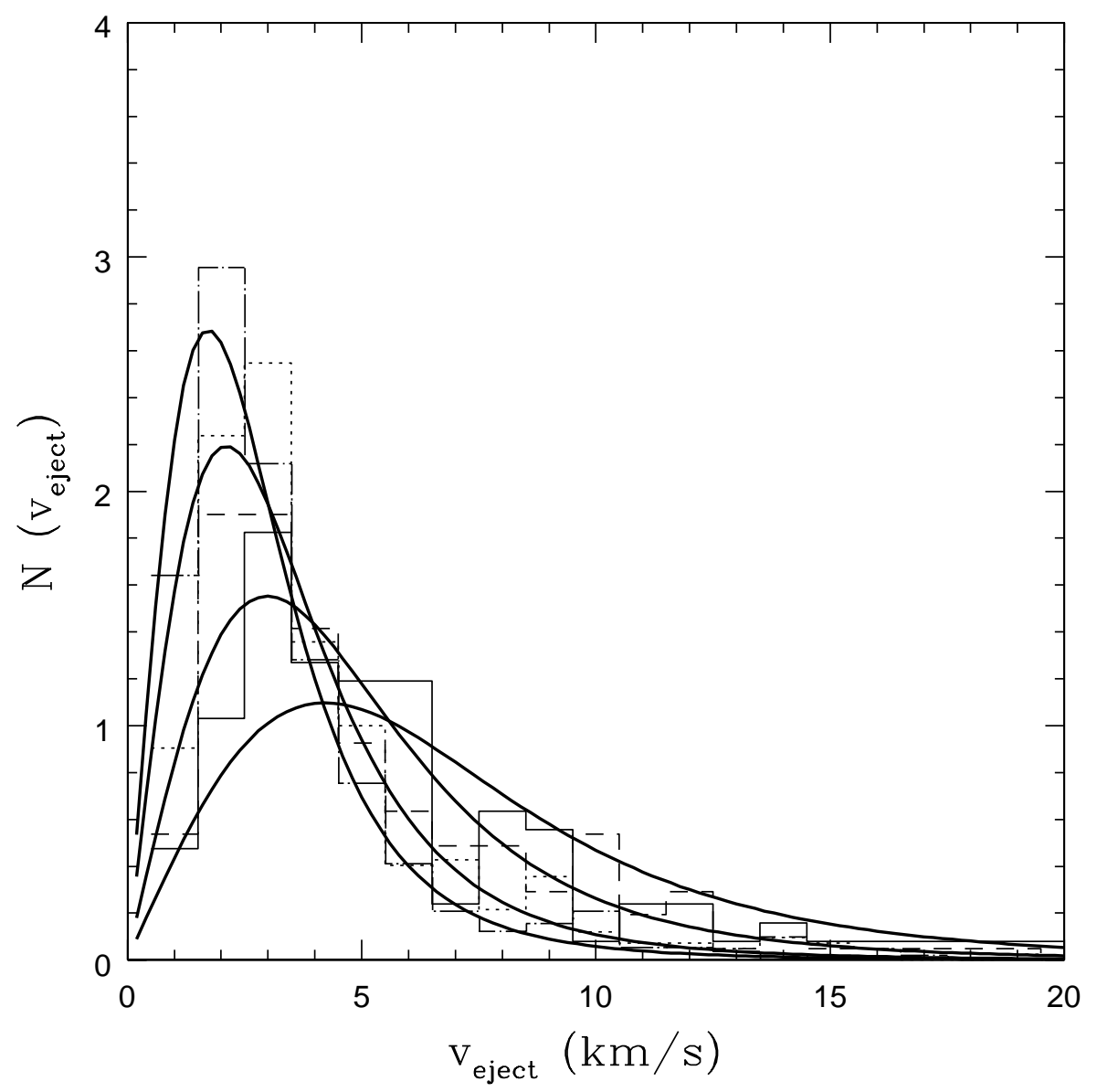

Fig. 12.- Distribution of ejection speeds for scattering interactions with central stellar mass $M_{*}$ $=0.50 M_{\odot}$. The histograms show the distributions of ejection speeds found in the numerical simulations for the analogs of Jupiter (solid), Saturn (dashes), Uranus (dots), and Neptune (dotdashes). The four smooth solid curves show the expected distribution from the simple theory outlined in the text. 


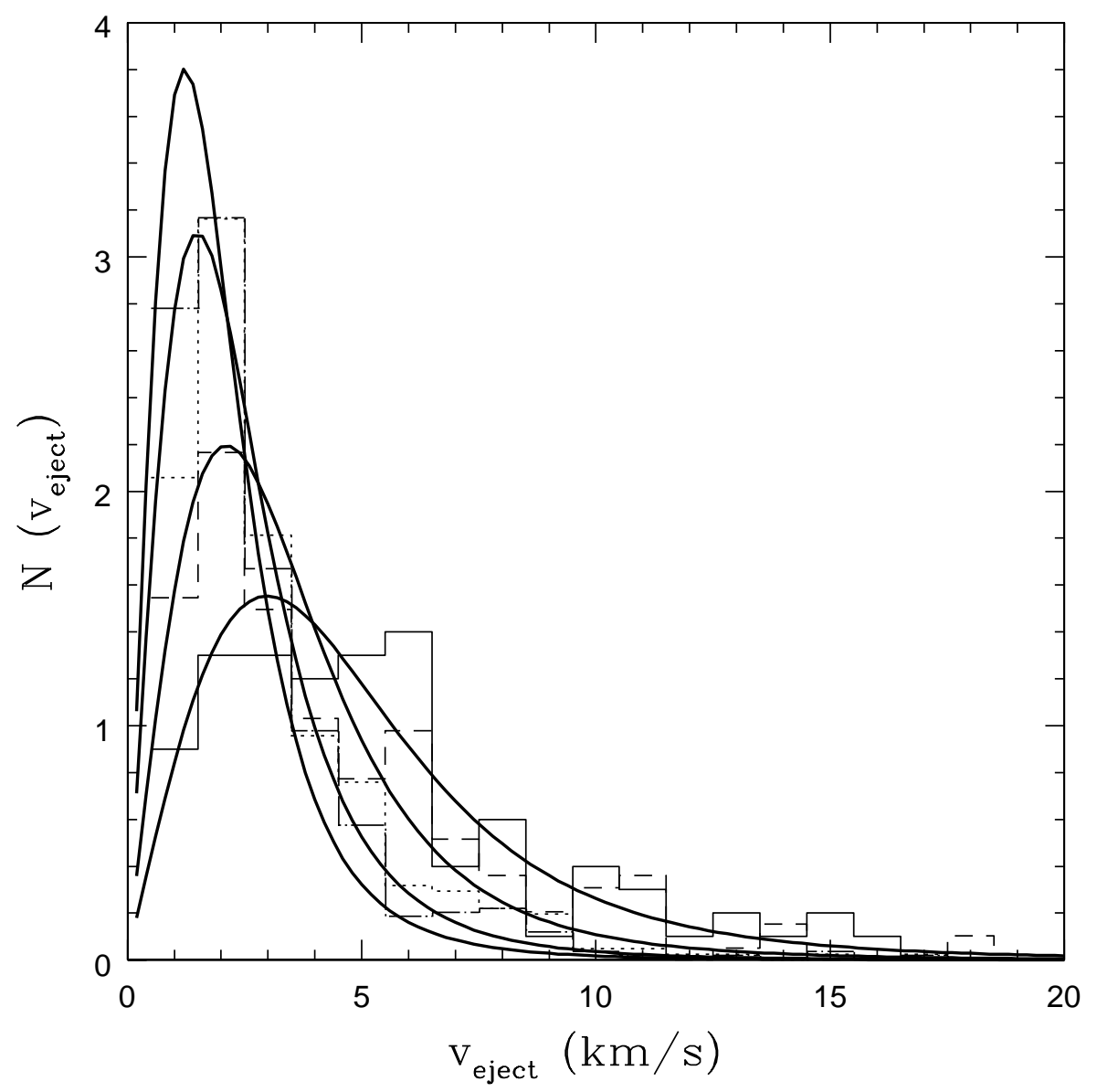

Fig. 13.- Distribution of ejection speeds for scattering interactions with central stellar mass $M_{*}$ $=0.25 M_{\odot}$. The histograms show the distributions of ejection speeds found in the numerical simulations for the analogs of Jupiter (solid), Saturn (dashes), Uranus (dots), and Neptune (dotdashes). The four smooth solid curves show the expected distribution from the simple theory outlined in the text. 
Another result from our ensemble of scattering experiments is the distribution of ejection speeds for planets that are removed from their solar systems during the interaction. The resulting distributions are shown for each of the four giant planets in Figure 12 (for stellar mass $M_{*}=0.50$ $\left.M_{\odot}\right)$ and Figure $13\left(M_{*}=0.25 M_{\odot}\right)$. Also shown are the theoretically expected distributions based on the idea that ejections involve sufficiently close encounters that the gravitational potential of the perturber (the passing star) dominates that of the central star. This type of interaction implies a distribution of ejection speeds of the basic form

$$
\frac{d p}{d u}=\frac{4 u}{\left(1+u^{2}\right)^{3}}
$$

where $u=v / v_{0}$ and the velocity scale is given by $v_{0}^{2}=G M_{*} / a$ (Moorhead \& Adams 2005). Notice that the impact speed of the binary $\left(v_{\text {inf }}\right)$ does not enter into this formula because $v_{0} \gg$

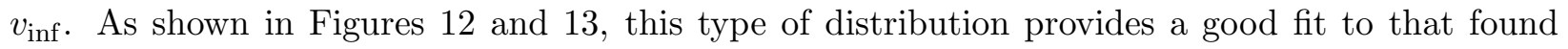
in the simulation data. Note that the numerical and analytic distributions are given the same normalization for all four planets. The overall number of ejections will vary with the planet's semimajor axes, as given by the cross sections in Tables $6-10$.

\section{NGC 1333 - A Case Study}

The recent identification of $93 \mathrm{~N}_{2} \mathrm{H}^{+}$(1-0) clumps in the young cluster NGC 1333 by Walsh et al. (2004ab) provides an excellent opportunity to apply the theoretical program developed in this paper toward the understanding of an observed cluster. More specifically, the data provide two dimensional (2D) position measurements (in the plane of the sky) and one dimensional (1D) velocity measurements (in the line of sight) for each of the 93 clumps. As a result, we need to reconstruct the remaining three phase space variables in order to make full three dimensional (3D) simulations of the cluster. Because the reconstruction process contains a random element (see below), we have to perform multiple realizations of the simulations in order to describe the dynamics. In addition, because the data do not completely specify the starting conditions (without reconstruction), this set of simulations represents a "theoretical model inspired by observations of NGC 1333" rather than a faithful model of the NGC 1333 cluster itself.

The starting conditions for the simulations are determined as follows. For a given $2 \mathrm{D}$ radius $r_{2 D}$ (as measured by Walsh et al. 2004ab), we use the fact that $r_{2 D}=\sin \theta r_{3 D}$, and assume that $\mu=\cos \theta$ is distributed randomly over the interval $[-1,1]$. This procedure allows us to reconstruct the missing spatial coordinate. The resulting radial mass profiles of the cluster are illustrated in Figure 14. The resulting mass profile is intermediate between that of an isothermal sphere with $M(r) \propto r$ and a less centrally densed profile with $M(r) \propto r^{2}$ (which corresponds to $\rho \sim r^{-1}$ as used in $\S 2)$. This particular cluster is thus somewhat more centrally concentrated than the theoretical models. In a similar manner, we assume that the (small) measured line of sight velocities are one component of an isotropic (small) 3D velocity vector, and reconstruct the missing velocity 
components accordingly. Since the observed speeds are small compared to the virial speeds, the starting conditions are much like the "cold" starting states of $\S 2$.

The observations also provide mass estimates for the clumps. For the sake of definiteness, we assume that each clump forms a star, and that the mass of the star is given by the mass of the clump. In actuality, the mass of the clump is not exactly given by the mass estimated from $\mathrm{N}_{2} \mathrm{H}^{+}$, as there is no hard boundary at the radius where the molecule becomes too faint to be seen; this effect makes the true clump masses larger than reported. On the other hand, we expect some inefficiency in the star formation process (e.g., Adams \& Fatuzzo 1996), which would make the stellar masses smaller than the clump masses. We are thus implicitly assuming that these two effects cancel out. The observations indicate that the clump masses in NGC 1333 are somewhat segregated, with the more massive clumps found near the cluster center. This trend is illustrated in Figure 15. In this regard, the simulations of NGC 1333 differ from the purely theoretical models of $\S 2$, where only minimal primordial mass segregation was included (the most massive star was placed at the cluster center). In addition to the mass in clumps, we include a smooth background potential of gas analogous to the gas component used in $\S 2$.

The results of the simulations for NGC 1333 are listed as the final entries in Tables 1, 2, and 3. As expected, the output parameters for this cluster are most like the theoretical clusters with $N=100$ and cold starting conditions. However, the NGC 1333 simulations produce clusters that are somewhat more concentrated and interactive. All of the indicators point in the same direction: Compared to $N=100$ simulations with cold starting states, the NGC 1333 simulations have half mass radii that are smaller by a factor of $\sim 1.8$, a somewhat larger fraction of stars that remain bound ( $69 \%$ versus $54 \%$ ), and a smaller isotropy parameter $\beta$ (see Table 1 ). The mass profiles have roughly the same scale length $r_{0} \approx 0.3-0.4 \mathrm{pc}$ (Table 2$)$, and a somewhat smaller index $(p=0.55$ compared to 0.69 ), indicating a more centrally concentrated cluster.

The largest difference between the NGC 1333 simulations and the others is reflected in the interaction rates, where the fiducial rate $\Gamma_{0}$ for NGC 1333 larger by a factor of $5-6$ (Table 3 ). This higher interaction rate is a direct result of the smaller half mass radius (a simple analytic approximation suggests that $\Gamma_{0} \sim R_{1 / 2}^{-7 / 2}$ ). The characteristic interaction distance $b_{C} \approx 238 \mathrm{AU}$, which implies that the NGC 1333 cluster facilitates disk truncation down to radii $r_{d} \sim 80$ AU (still well outside the realm of the giant planets in our solar system). Planetary analogs of Neptune can be stripped from the smaller stars with $M_{*}=0.25 M_{\odot}$ and can experience large eccentricity enhancements $(e \sim 0.7)$ when orbiting solar type stars (see Tables $6-11)$. Planetary analogs of Jupiter remain largely unperturbed around all stars. This level of disruption is somewhat higher than found earlier for the purely theoretical clusters, but still remains modest.

One important lesson resulting from this case study of NGC 1333 is the extent to which initial conditions can affect forming planetary systems. Compared to the starting conditions used for the simulations in $\S 2$ (where these starting conditions were motivated by observational surveys of cluster conditions, e.g., Figs. 1 and 2), the NGC 1333 starts with a higher degree of central 


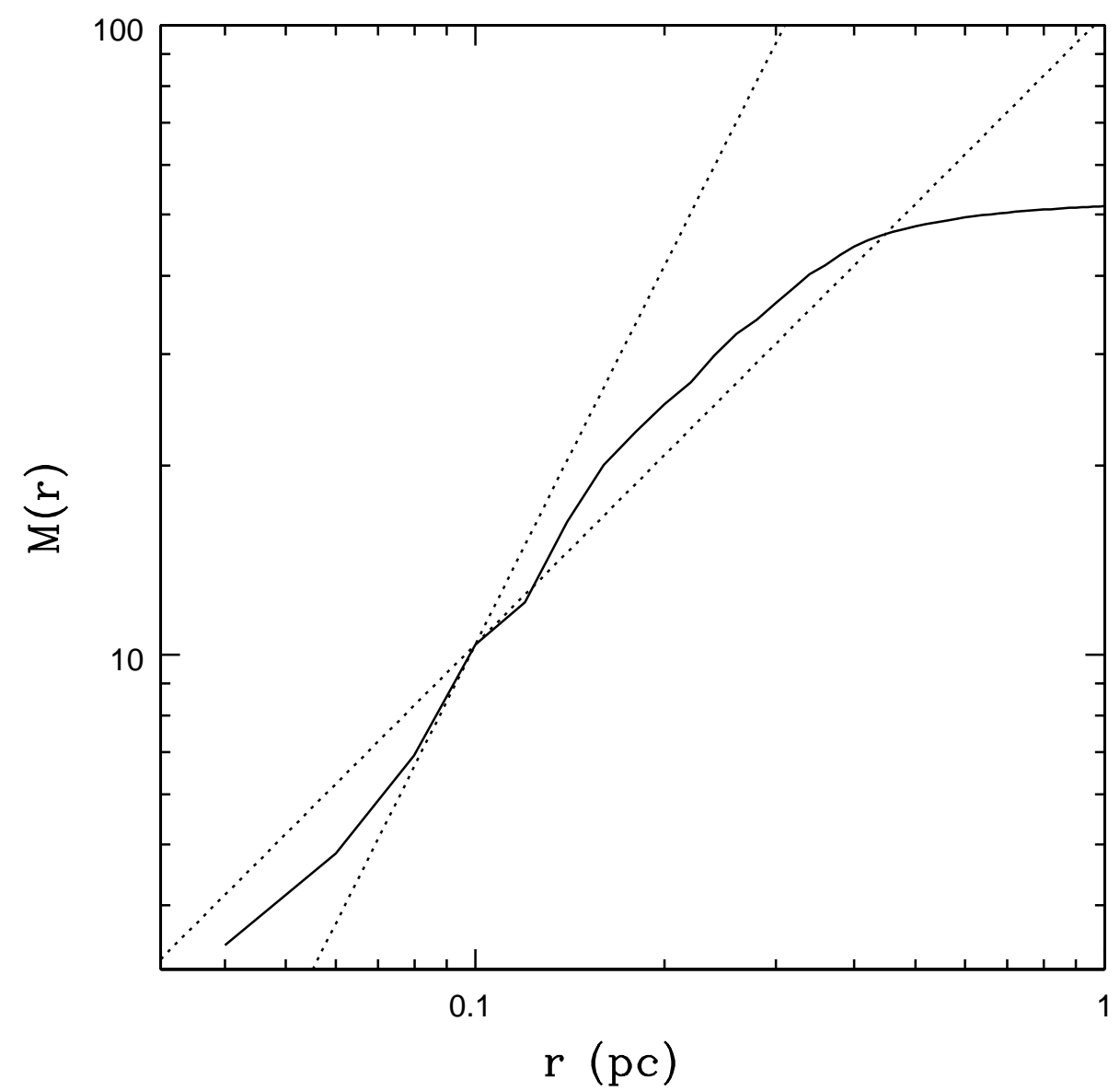

Fig. 14.- Reconstructed radial mass profile of the young embedded cluster NGC 1333, where $M(r)$ is given in $M_{\odot}$. To obtain this profile, the two dimensional observational map (Walsh et al. 2004ab) is converted into $10^{5}$ different realizations of the three dimensional cluster structure (and averaged) according to the procedure outlined in the text. The two dotted lines, included for reference, have power-law slopes $p=1$ and 2, i.e., $M(r) \propto r$ (which corresponds to $\rho(r) \propto r^{-2}$ ) and $M(r) \propto r^{2}$ (which corresponds to $\rho(r) \propto r$ ). 


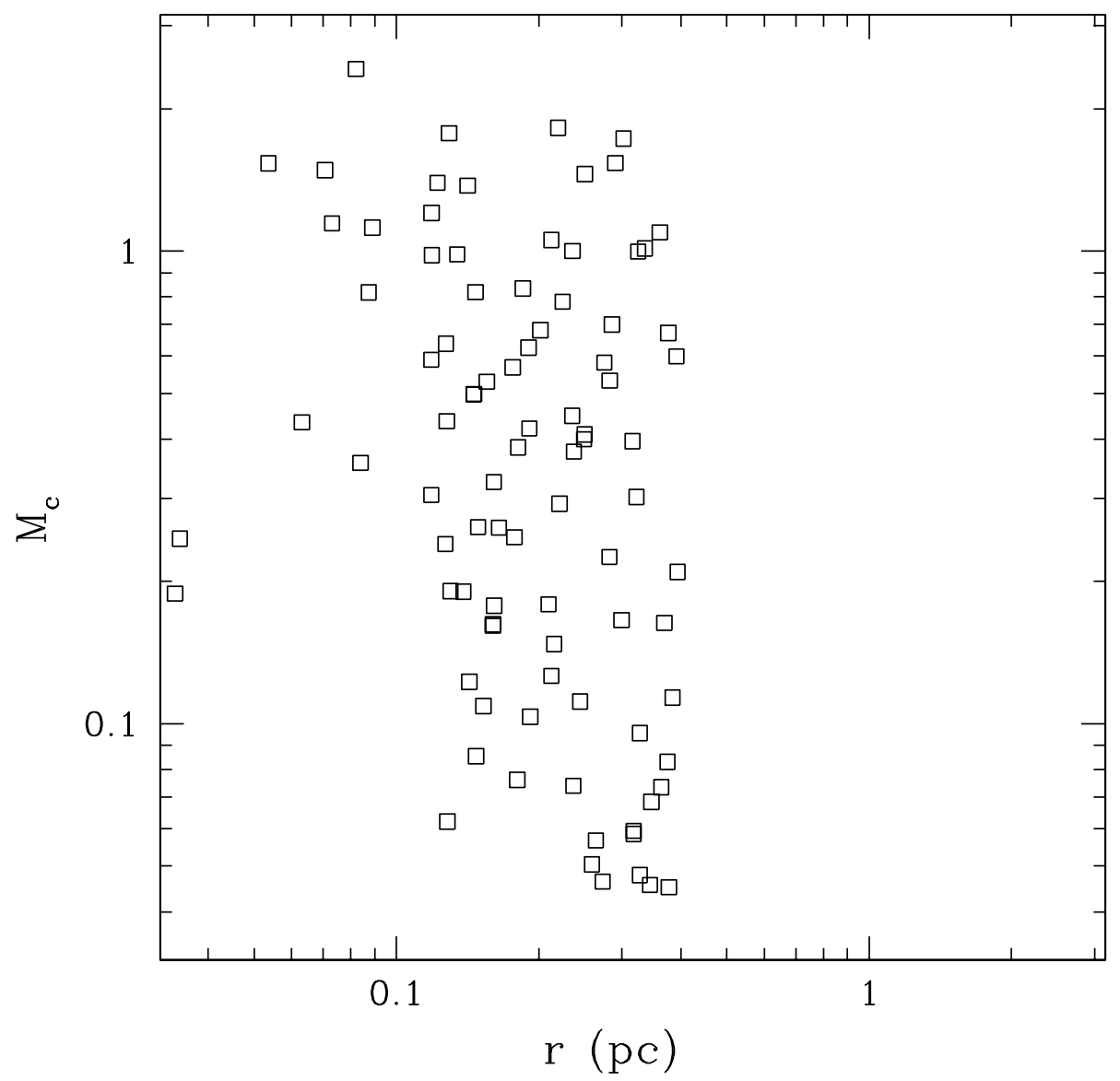

Fig. 15.- Mass $M_{c}$ of observed clumps (in $M_{\odot}$ ) as a function of two dimensional radius for the young embedded cluster NGC 1333. The data are taken from Walsh et al. (2004ab). Notice that the primordial mass segregation in this system is somewhat greater than the minimal segregation used in the purely theoretical models (§2). 
concentration and a greater amount of primordial mass segregation. The result is a more compact cluster (smaller $R_{1 / 2}$ ) and hence a higher interaction rate. In addition, the cold starting condition allows stars to fall inside much of the original gas and this geometry enhances cluster survival after gas removal (compare Adams 2000 with Geyer \& Burkert 2001). In order to fully determine the effects of the cluster environment on forming solar systems, we need to determine the range of starting density profiles and mass segregation.

\section{Conclusion}

This paper has explored the early dynamical evolution of embedded stellar groups and clusters with stellar membership in the range $N=100-1000$. This work includes $N$-body simulations of the dynamics, compilations of the distributions of FUV luminosities and fluxes, the calculation of scattering cross sections for young planetary systems, and an application to the observed embedded cluster NGC 1333. Our main conclusion is that clusters (with the range of properties considered here) have relatively modest effects on star and planet formation. The interaction rates and radiation levels are low, so that forming stars and their accompanying planetary systems are largely unperturbed by their environment. This finding, in turn, implies that cluster structure is due primarily to the initial conditions, rather than interactions. These results can be summarized in greater detail as follows:

[1] In order to obtain good statistics for our output measures, we have performed 100 realizations of each set of initial conditions for groups/clusters with $N=100,300$, and 1000. In addition to considering different cluster sizes $N$, we consider both "virial" and "cold" initial conditions. These simulations show a significant difference between the two types of starting conditions. Compared with "virial" initial conditions (near virial equilibrium), "cold" clusters are more centrally concentrated, retain more of their stars for longer times, and exhibit more radial velocity distributions (Tables 1 and 4). As expected, all clusters lose stars and gradually spread out with time. This behavior is quantified by finding the average time evolution of each group/cluster type using 100 realizations of each set of initial conditions (Fig. 3 and Table 1). We also provide quantitative descriptions of these systems by finding the mass profiles of the clusters (Fig. 4, eq. [3], and Table 2) and the distributions of close encounters (Fig. 5, eq. [5], and Table 3). All of these quantities can be used in a variety of other contexts to test further the effects of the cluster environment on the processes of star and planet formation.

[2] We have calculated the FUV radiation expected from this class of groups and clusters. This issue involves (at least) three separate distributions: Clusters of a given size $N$ display a wide distribution of FUV luminosities due to incomplete sampling of the stellar IMF; we have determined this distribution $P\left(L_{F U V}\right)$ as a function of $N$ (Figs. 6 and 7). Clusters themselves come in a distribution of sizes $P(N)$ (Fig. 1) and we have found the distribution of FUV luminosities sampled over all clusters (Fig. 8) using the observed range of cluster radii $R$ (Fig. 2). Finally, the stars within a cluster explore a range of radial positions, which in turn specify the distribution 
of radial positions $P(r)$ in the cluster. These three probability distributions $\left[P(N), P\left(L_{F U V}\right)\right.$, $P(r)$ ] jointly determine the composite distribution of FUV fluxes that impinge upon the composite ensemble of forming solar systems (shown in Fig. 9). The median FUV flux for the composite distribution is only $G_{0} \approx 900$, which is not intense enough to evaporate disks orbiting solar-type stars (over $10 \mathrm{Myr}$ ) for the range of radii of interest for planet formation ( $r \leq 30 \mathrm{AU}$ ). We have also found the fluxes averaged over individual orbits within the clusters as a function of orbital energy and angular momentum (eqs. [20 - 24]). The results of this section imply that FUV radiation in clusters does not generally inhibit planet formation. In addition, the distributions found here can be used to determine the radiation exposure for forming solar systems in a variety of other contexts.

[3] We have calculated the cross sections for the interaction of newly formed solar systems with passing binaries (Tables $6-11$ ) using an ensemble of $\sim 10^{5}$ Monte Carlo scattering experiments. These cross sections, in conjunction with the interaction rates determined via the $N$-body simulations, show that the typical solar system is not greatly affected by scattering interactions within its birth aggregate. The "typical" star within a cluster of size $N=100-1000$ will experience approximately one close encounter within a distance $b_{C}$ over a $10 \mathrm{Myr}$ window of time. We find that $b_{C}=700-4000 \mathrm{AU}$ for the systems considered here. This passage is not close enough to appreciably enhance the eccentricity of Neptune in our solar system. The mildest disruption event considered here is the increase in eccentricity of a Neptune-analog planet orbiting a $0.125 M_{\odot}$ star; the cross section for this event is $\langle\sigma\rangle \sim 2 \times 10^{5} \mathrm{AU}^{2}$ (Table 10), requiring a closest approach distance of $\sim 250$ AU. Similarly, disks are truncated by passing stars down to radii of $\sim 1 / 3$ of the closest approach distance (Kobayashi \& Ida 2001), so the disks in these clusters are expected to be limited to 230 - $1300 \mathrm{AU}$, much larger than the regimes of interest for planet formation. Our main conclusion is that planet forming disks and newly formed solar systems generally survive their birth aggregates with little disruption. In addition, the cross sections calculated herein can be used to study solar system disruption in a wider range of contexts and environments. For example, planet formation can potentially be induced by weak scattering encounters (Thies et al. 2005). As another application, we note that some star forming regions are reported to have higher binary fractions than the field. As a result, one issue is whether or not the cluster environment can disrupt binaries (e.g., Kroupa et al. 2003 and references therein). Our results imply that the clusters considered here do not facilitate the disruption of binaries, except for those that begin with separations greater than $1000 \mathrm{AU}$. If the primordial period distribution is similar to that measured in the field, only $\sim 1 / 7$ of binaries would be affected by scattering interactions (of course, more binary disruption would occur if these clusters did not suffer an early demise due to gas expulsion at $t=5 \mathrm{Myr}$ ).

[4] We have performed an ensemble of 100 simulations of the observed young embedded cluster NGC 1333, where we start with observed positions in the plane of the sky and line of sight velocity components, and then reconstruct the remaining phase space coordinates. This set of simulations is used to construct the output measures for clusters of this type and we use the results to assess the impact of the background environment on star and planetary sytems forming within this type of group/cluster. This cluster is most like the $N=100$ cold simulations performed in $\S 2$. Compared 
to the purely theoretical simulations, NGC 133 has more primordial mass segregation and a smaller half-mass radius $R_{1 / 2}$. This property leads to a somewhat larger bound fraction $f_{b}$ and a higher interaction rate $\Gamma$ compared to the $N=100$ simulations with cold starts. Nonetheless, the overall amount of disruption is small (e.g., circumstellar disks are truncated down to $\sim 80 \mathrm{AU}$, well outside the region where giant planets form) so that the cluster environment has only a modest effect on star and planet formation.

This paper represents an assessment of dynamical effects in six classes of young embedded clusters. The treatment is comprehensive in that we run $100 \mathrm{~N}$-body simulations for each type of cluster in order to obtain robust statistical descriptions, and we assess the effects of FUV radiation and solar system scattering on forming solar systems. On the other hand, the parameter space available to such clusters is enormous and a great deal of additional work remains to be done. For example, the simulations in this study were started with cluster sizes $R_{c *}$ near the low end of the observed range (the lower dashed curve in Fig. 2) and gas removal times (5 Myr) near the high end of the observed range (Lada \& Lada 2003). These choices tend to make the clusters denser and long-lived, which makes the effects of interactions and radiation more important. Since we find that interactions and radiation have only modest effects on planet forming disks, we can consider this conclusion as conservative. However, a more detailed treatment of gas removal, including shorter lifetimes and more realistic time dependence (not a step function in time), is warranted.

A number of additional processes may also affect cluster evolution and should be studied; these include the role played by additional primordial mass segregation (beyond the minimal treatment used here), non-spherical starting conditions for both the stars and gas, and the effects of primordial binaries. Mass segregation - both primordial and evolutionary - may be particularly interesting, as suggested by our simulations motivated by NGC 1333. Our work to date indicates that the disruption of planetary systems is a sensitive function of the mass $M_{*}$ of the central star (e.g., scattering cross sections scale approximately as $\langle\sigma\rangle \sim M_{*}^{-1 / 2}$ and FUV radiation truncates disks approximately at $r_{d} \sim M_{*}$ ) and a sensitive function of location within the cluster (both the FUV flux and interaction rates are much greater near the cluster center). If substantial mass segregation is present during the $\sim 10 \mathrm{Myr}$ while the clusters remain intact, the larger stars will be closer to the center where they are exposed to greater probability of disruption, and the smaller stars will be farther out and relatively safer. The degree to which this effect occurs should be quantified in future studies.

Finally, this work emphasizes the fact that cluster environments display a distribution of properties and the full distributions must be considered in order to assess their effects on forming stars and planets. Some previous studies (e.g., Bonnell \& Bate 2002) have focused on the densest regions of large clusters where the interaction rates are high and the background environment has an important effect on star formation. Although most clusters have a central zone of high interaction, for the clusters considered here most stars do not live in the highly interactive zone. It is thus crucial to determine the full distribution of environmental properties that forming stars are exposed to, including how often the various environments arise. Clusters are sampled from a 
distribution of sizes $P(N)$. For a given size $N$, clusters have a range of radial sizes $P\left(R_{c *}\right)$, a range of starting speeds and hence a distribution of virial parameters $P(Q)$, and display a distribution of FUV luminosities $P\left(L_{F U V}\right)$. For given sizes $R_{c *}$ and $N$, and a given starting condition $Q$, stellar members explore a distribution of radial positions $P(r)$ within the cluster. The methods developed in this paper show that we can find the distributions of luminosities (Fig. 8), radial positions (analogous to Fig. 4), closest approaches (Fig. 5), and other quantities of interest from a given set of starting conditions. Perhaps the most important goal of future studies is thus to make a better observational determination of the distributions $P(N), P(Q)$, and $P\left(R_{c *}\right)$, which would allow for a more complete assessment of the effects of the cluster environment on star and planet formation.

\section{Acknowledgment}

We would like Lori Allen, Tony Bloch, Gus Evrard, and Tom Megeath for many beneficial discussions. This work was supported at the University of Michigan by the Michigan Center for Theoretical Physics, and by NASA through the Terrestrial Planet Finder Mission (NNG04G190G) and the Astrophysics Theory Program (NNG04GK56G0). This work was supported at Xavier University through the Hauck Foundation. Finally, P. Myers acknowledges Grant NAG5-13050 from the NASA Origins of Solar Systems Program. 


\section{REFERENCES}

Aarseth, S. J. 1999, PASP, 111, 1333

Aarseth, S. J. 2001, NewA, 6, 277

Aarseth, S. J. 2003, Gravitational N-Body Simulations (Cambridge: Cambridge Univ. Press)

Adams, F. C. 2000, ApJ, 542, 964

Adams, F. C., \& Bloch, A. M. 2005, ApJ, 629, 204 (AB05)

Adams, F. C., \& Fatuzzo, M. 1996, ApJ, 464, 256

Adams, F. C., Hollenbach, D., Laughlin, G., \& Gorti, U. 2004, ApJ, 611, 360

Adams, F. C., \& Laughlin, G. 2001, Icarus, 150, 151

Adams, F. C., \& Myers, P. C. 2001, ApJ, 553, 744

André, P. 2002, Astro. Sp. Sci., 281, 51

Armitage, P. J. 2000, A\&A, 362, 968

Baumgardt, H., Hut, P., \& Heggie, D. C. 2002, MNRAS, 336, 1069

Binney, J., \& Tremaine, S. 1987, Galactic Dynamics, (Princeton: Princeton Univ. Press), (BT87)

Boily, C. M., \& Kroupa, P. 2003a, MNRAS, 338, 665

Boily, C. M., \& Kroupa, P. 2003b, MNRAS, 338, 673

Bonnell, I. A., \& Bate, M. R. 2002, MNRAS, 336, 659

Bonnell, I. A., Clarke, C. J., Bate, M. R., \& Pringle, J. E. 2001, MNRAS, 324, 573

Bonnell, I. A., \& Davies, M. B. 1998, MNRAS, 295, 691

Carpenter, J. M. 2000, AJ, 120, 3139

Carpenter, J. M., et al. 1997, AJ, 114, 198

David, E. M., Quintana, E. V., Fatuzzo, M., \& F. C. Adams 2003, PASP, 115, 825

de la Fuente Marcos, C., \& de la Fuente Marcos, R. 1997, A\&A, 326, 21

de la Fuente Marcos, C., \& de la Fuente Marcos, R. 1999, NewA, 4, 21

Duquennoy, A., \& Mayor, M. 1991, A\&A, 248, 485 
Elmegreen, B. G. 1991, The Physics of Star Formation and Early Evolution, eds. Lada, C. J. \& Kylafis, N. D., (Dordrecht: Kluwer), p. 35

Elmegreen, B. G., Efremov, Y., Pudritz, R. E., \& Zinnecker, H. 2000, Protostars and Planets IV, eds. Mannings V., Boss, A. P., \& Russell, S. S., (Tucson: Univ. Ariz. Press), p. 179

Fatuzzo, M., Adams, F. C., \& Myers, P. C. 2004, ApJ, 615, 813

Fregeau, J. M., Chatterjee, S., \& Rasio, F. A. 2005, submitted to ApJ, astro-ph/0510748

Geyer, M. P., \& Burkert, A. 2001, MNRAS, 323, 988

Giersz, M., \& Heggie, D. C. 1994, MNRAS, 268, 257

Goodman, J., Heggie, D. C., \& Hut, P. 1993, ApJ, 415, 715

Gutermuth, R. A., et al. 2004, ApJS, 154, 374

Gutermuth, R. A., et al. 2005, ApJ, 632, 397

Heggie, D. C., Hut, P., \& McMillan, S. L. W. 1996, ApJ, 467, 359

Heller, C. H. 1993, ApJ, 408, 337

Heller, C. H. 1995, ApJ, 455, 252

Henry, T. J. 1991, PhD Thesis, University of Arizona, Tucson

Hernquist, L. 1990, ApJ, 356, 359

Hillenbrand, L. A., \& Hartmann, L. 1998, ApJ, 492, 540

Hurley, J. R., \& Shara, M. M. 2002, ApJ, 565, 1251

Hut, P., \& Bahcall, J. N. 1983, ApJ, 268, 319

Ida, S., Larwood, J., \& Burkert, A. 2000, ApJ, 528, 351

Jiang, Z. B., et al. 2002, ApJ, 577, 245

Jijina, J., Myers, P. C., \& Adams, F. C. 1999, ApJS, 125, 161

Johnstone, D., Hollenbach, D. J., \& Bally, J. 1998, ApJ, 499, 758

Klessen, R. S., \& Burkert, A. 2000, ApJS, 128, 287

Klessen, R. S., \& Burkert, A. 2001, ApJ, 549, 386

Klessen, R. S., Heitsch, F., \& Mac Low, M-M. 2000, ApJ, 535, 887 
Kobayashi, H., \& Ida, S. 2001, Icarus, 153, 416

Kroupa, P. 1995a, MNRAS, 277, 1507

Kroupa, P. 1995b, MNRAS, 277, 1522

Kroupa, P., Aarseth, S., \& Hurley, J. 2001, MNRAS, 321, 699

Kroupa, P., \& Bouvier, J. 2003, MNRAS, 346, 343

Kroupa, P., Bouvier, J., Duchene, G., Moraux, E. 2003, MNRAS, 346, 354

Kroupa, P., Petr, M. G., \& McCaughrean, M. J. 1999, NewA, 4, 495

Lada, C. J., \& Lada, E. A. 2003, ARA\&A, 41, 57

Lada, C. J., Margulis, M., \& Dearborn, D. 1984, ApJ, 285, 141

Larson, R. B. 1985, MNRAS, 214, 379

Laughlin, G., \& Adams, F. C. 2000, Icarus, 145, 614

Laughlin, G., Bodenheimer, P., \& Adams, F. C. 2004, ApJ, 612, 73

Leisawitz, D., Bash, F., \& Thaddeus, P. 1989, ApJS, 70, 731

Maeder, A., \& Meynet, G. 1987, A\&A, 182, 243

Massey, P. 2003, ARA\&A, 41, 15

Matzner, C. D., \& McKee, C. F. 2000, ApJ, 545, 364

McKee, C. F., \& Tan, J. C. 2003, ApJ, 585, 850

McMillan, S. L. W., \& Hut, P. 1996, ApJ, 467, 348

Moorhead, A. V., \& Adams, F. C. 2005, Icarus, 178, 517

Myers, P. C. 1998, ApJL, 496, L109

Myers, P. C., \& Fuller, G. A. 1993, ApJ, 402, 635

Ostriker, E. C. 1994, ApJ, 424, 292

Peretto, N., Andre, Ph., \& Belloche, A. 2005, A\&A, in press, astro-ph/0508619

Porras, A., et al. 2003, AJ, 126, 1916

Portegies Zwart, S. F., Hut, P., Makino, J., \& McMillan, S.L.W. 1998, A\&A, 337, 363 
Press, W. H., Teukolsky, S. A., Vetterling, W. T., \& Flannery, B. P. 1986, Numerical Recipes: The art of scientific computing (Cambridge: Cambridge Univ. Press)

Rasio, F. A., McMillan, S., \& Hut, P. 1995, ApJ, 438, 33

Richtmyer, R. D. 1978, Principles of Advanced Mathematical Physics (New York: Springer-Verlag)

Salpeter, E. E. 1955, ApJ, 121, 161

Scally, A., \& Clarke, C. 2001, MNRAS, 325, 449

Schaller, G., Schaerer, D., Meynet, G., \& Maeder, A. 1992, A\&AS, 96, 269

Shu, F. H. 1977, ApJ, 214, 488

Shu, F. H., Johnstone, D., \& Hollenbach, D. J. 1993, Icarus, 106, 92

Smith, K. W., \& Bonnell, I. A. 2001, MNRAS, 322, L1

Sterzik, M. F., \& Durisen, R. H. 1998, A\&A, 339, 95

Störzer, H., \& Hollenbach, D. 1999, ApJ, 515, 688

Testi, L., Palla, F., \& Natta, A. 1998, A\&AS, 133, 81

Testi, L., Palla, F., \& Natta, A. 1999, A\&A, 342, 515

Thies, I., Kroupa, P., \& Theis, C. 2005, astro-ph/0510007

Walsh, A. J., et al. 2004a, poster presented at the CDJO2004 meeting in Banff, July 12 - 16

Walsh, A. J.., Myers, P. C., \& Burton, M. G. 2004b, ApJ, 614, 194

Whitworth, A. 1979, MNRAS, 186, 59 